\title{
Review \\ Ultrasonic Treatment as the Route for Grain Refinement of Mg-Al Alloys: A Systematic Review
}

\author{
Inês V. Gomes ${ }^{1,2, *}$, Hélder Puga ${ }^{2} \mathbb{D}$ and José L. Alves ${ }^{2}$ \\ 1 MIT Portugal Program-School of Engineering, University of Minho, 4800-058 Guimarães, Portugal \\ 2 CMEMS-Center for Micro-Electro-Mechanical Systems, University of Minho, 4800-058 Guimarães, Portugal; \\ puga@dem.uminho.pt (H.P.); jlalves@dem.uminho.pt (J.L.A.) \\ * Correspondence: inesvarela@dem.uminho.pt
}

Citation: Gomes, I.V.; Puga, H.;

Alves, J.L. Ultrasonic Treatment as the Route for Grain Refinement of Mg-Al Alloys: A Systematic Review. Metals 2021, 11, 1529. https://doi.org/ $10.3390 /$ met11101529

Academic Editor: Daolun Chen

Received: 1 September 2021

Accepted: 21 September 2021

Published: 26 September 2021

Publisher's Note: MDPI stays neutral with regard to jurisdictional claims in published maps and institutional affiliations.

Copyright: (c) 2021 by the authors. Licensee MDPI, Basel, Switzerland. This article is an open access article distributed under the terms and conditions of the Creative Commons Attribution (CC BY) license (https:// creativecommons.org/licenses/by/ $4.0 /)$.

\begin{abstract}
In this work, a systematic review of the published literature was conducted, following the guidelines of the Preferred Reporting Items for Systematic Reviews and Meta-Analyses, on the ultrasonic treatment of magnesium-aluminium alloys for grain refinement. Scopus, Science Direct, and Web of Science databases were used in the literature search, which was finished by the 1st of June 2021. Seventeen articles met the eligibility criteria and were considered in this review, organized according to the type of ultrasonic treatment applied: isothermal (8/17) or continuous $(9 / 17)$. Summary tables were used to categorize the information gathered from the articles, namely Treatment Conditions, Microstructural and Mechanical Analysis, and Mechanisms Behind Ultrasonic Grain Refining Ability. This systematic review aimed to structure and organize the available information regarding the ultrasonic processing of magnesium-aluminium alloys so new researchers can find a start point for their works and identify potential gaps in this research field.
\end{abstract}

Keywords: ultrasonic melt processing; grain refinement; magnesium alloys; mechanical properties; systematic review

\section{Introduction}

Interest in magnesium and its alloys has been rising in recent years, especially for structural applications in the automotive, railway, and aerospace industries [1-3]. Such popularity stems from an engaging combination of comprehensive properties, namely low density, high strength-to-weight ratio, excellent machinability, and good castability [4-6]. Moreover, magnesium is considered as the best green material of the 21st century, which yields an increasing interest in studying it comprehensively more recently [7].

Despite the attractive characteristics of this material, demanding requirements are imposed on the quality of the products, which stresses continuous improvement of the metal-processing technologies [8]. In addition, heterogeneous and coarse dendritic morphology and its high susceptibility to solidification defects are detrimental to magnesium's mechanical properties [9].

Several authors have pointed the microstructure refinement of cast parts as a route for overcoming the material defects enhancing magnesium alloys' mechanical performance [5-10]. Moreover, casting technologies that may provide a fine and homogeneous microstructure can be of great value since there is a great demand for economic techniques capable of improving the downstream manufacturing processes, which can be valuable for industrial application [11]. The grain refinement of aluminium-free magnesium alloys can be easily achieved by adding elements such as zirconium, and some works have already been carried out using additions between $0.2 \%-1.0 \mathrm{wt} . \%$ to promote heterogeneous nucleation [12-16].

However, this approach does not produce satisfactory results in magnesium-aluminium alloys due to the initial formation of stable intermetallic phases between aluminium and zirconium such as $\mathrm{Al}_{3} \mathrm{Zr}$, which are ineffective as nucleants for magnesium grains [17-19]. 
Likewise, refining elements like carbon, calcium, and strontium may also be added. Still, the formation of intermediate compounds brings environmental problems [20], hot tearing [21], and reduced ambient temperature properties [22], and therefore the results have not been promising [23].

The matrix microstructure modification by a physical process may overcome the limitation of chemical refinement methods as it may be applied regardless of the alloy composition and without changing it [6]. In the physical route to grain refinement, an external field such as electromagnetic or mechanical stirring/vibration, pulse electric current, intensive shearing using twin screw, among others, is introduced into the molten material during its solidification [23]. However, some hindrances were found regarding the application of some of these methods. The application of mechanical stirring technique is associated with the formation of unpredictable microstructures and phases due to the high temperature of the melt that usually compromises the stirrer integrity [24]. On the other hand, in the electric current pulse technology, the electric pulse needs to be directly passed through the melt, which may lead to its contamination, while pulsed magnetic field requires an extremely high current which can result in undesirable splashing at the top surface of the melt $[25,26]$.

In this scope, ultrasonic treatment has demonstrated promising results in refining magnesium-aluminium alloys $[6,27]$, which is the reason why the number of studies focused on this topic has been increasing in recent years.

When compared with other processing technologies for grain refinement, this technique presents significant advantages concerning its industrial application, namely high grain-refining efficacy, ease of operation, and affordable cost, requiring a quite simple apparatus. Moreover, conversely to what is verified in aluminium alloys' melts, titanium sonotrode, which provides excellent dimensional stability and high efficiency in transmitting the ultrasonic vibration, is very stable and almost insoluble in magnesium alloys, preventing melt contamination $[28,29]$. Another advantage of ultrasonication is that melt poisoning by oxidation is significantly diminished during irradiation, given that the melt surface is not severely disturbed.

In practice, the ultrasonic energy may be isothermal or continuously introduced directly into the melt through an immersed sonotrode. A non-contact approach, a designated indirect one, may be preferred depending on the melting point and chemical reactivity of the sonotrode material [27]. The mechanisms under the microstructure modification depend on which kind of treatment is applied, and therefore differences between results are expected. Indeed, the results obtained are conditioned by a significant number of factors, including the temperature at which the treatment is applied, its duration, and power.

Although some valuable review articles about liquid metal processing and solidification engineering, such as the one from Czerwinski [30], have already been published, this paper presented a systematic review of the work published in the scope of ultrasound application for grain refinement of magnesium-aluminium alloys, given the interest aroused by these materials for high-tech applications. In this way, the main goal was to summarize and discuss the work developed by the authors regarding this subject, especially their methods and results, so further studies may raise from the potential gaps found under this research topic.

\section{Methods}

The present systematic review was conducted according to the Preferred Reporting Items for Systematic Reviews and Meta-Analyses (PRISMA) guidelines.

\subsection{Search Strategy}

A search was performed until the 1st of June 2021, and no publication year limit was applied. The keywords "magnesium alloys", "grain refinement", "ultrasound", "melt treatment", "casting", and "mechanical properties", combined with the Boolean operators AND, OR, and NOT were used for search in Scopus, ScienceDirect, and Web of Science databases. 


\subsection{Study Selection}

All records were extracted to an Excel file, and duplicates were removed by the software filter and manually checked. The reference list of relevant articles identified through database search was analyzed so other potentially interesting studies could be considered for addition to this review.

After title and abstract screening, the records that (1) were from conference proceedings, (2) were abstract commentaries, review articles, or encyclopedia chapters, (3) were studies about aluminium-free magnesium alloys, (4) applied other manufacturing technologies than gravity casting, (5) used ultrasound energy combined with solute addition, or (6) were focused on composites materials were excluded. Finally, the selected articles were retrieved, and the respective full texts were carefully analyzed for eligibility.

\subsection{Data Collection and Extraction}

Data extraction tables were designed to organize critical details from each study to compare methodologies and results. The following information was gathered after the articles were classified in isothermal or continuous treatment: (i) alloy and treatment parameters, (ii) microstructural characteristics, (iii) mechanical properties before and after the ultrasonic treatment, (iv) mechanisms that the authors propose as underlying the grain refinement results obtained using the ultrasonic treatment, and (v) key findings of the studies considered. When only partial information was described in the original study, references and authors' previous works were analyzed to provide comparable data across the selected studies.

\section{Results}

Database and manual search provided 827 references. After duplicates removal, 303 titles and abstracts were screened, and 23 full-texts were considered for detailed analysis. Seventeen studies met the eligibility criteria and were considered in this systematic review. The systematic research methodology followed in this work is presented in Figure 1. Table 1 lists the selected articles titles, authors, and year of publication.

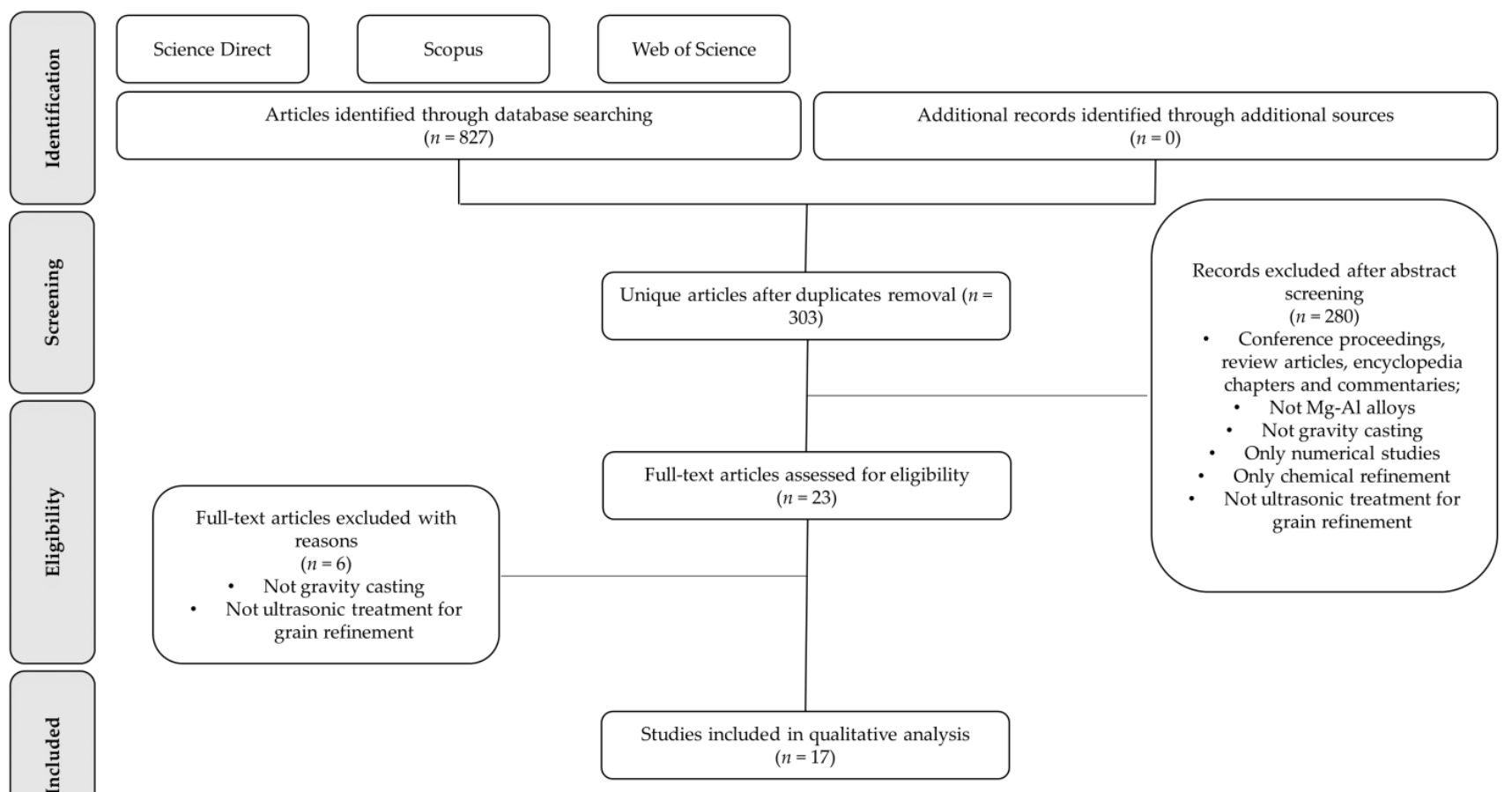

Figure 1. Flowchart of the search strategy conducted in this systematic review. 
Table 1. Articles included in the review.

\begin{tabular}{|c|c|}
\hline Reference & Title \\
\hline [27] & Effects of ultrasonic treatment on microstructures of AZ91 alloy \\
\hline [3] & $\begin{array}{l}\text { The Influence of High-Temperature Ultrasonic Processing Time on the Microstructure and Mechanical Properties } \\
\text { AZ91E Magnesium Alloy }\end{array}$ \\
\hline [6] & Effect of Ultrasonication on the Solidification Microstructure in $\mathrm{Al}$ and Mg-Alloys \\
\hline [8] & Effects of ultrasonic treatment on microstructure and tensile strength of AZ91 magnesium alloy \\
\hline [9] & Microstructural evolution in ultrasonicated AS41 magnesium alloy \\
\hline [10] & Grain refinement of AZ91 alloy by introducing ultrasonic vibration during solidification \\
\hline [28] & Ultrasonic refinement of magnesium by cavitation: Clarifying the role of wall crystals \\
\hline [31] & Microstructure and mechanical properties of AZ91 alloy produced with ultrasonic vibration \\
\hline [32] & Potency of high-intensity ultrasonic treatment for grain refinement of magnesium alloys \\
\hline [33] & Effect of ultrasonic power on microstructure and mechanical properties of AZ91 alloy \\
\hline [34] & The Effect of Ultrasonic Treatment on Microstructural and Mechanical Properties of Cast Magnesium Alloys \\
\hline [35] & Effect of ultrasonic vibration and solution heat treatment on microstructures and tensile properties of AZ91 alloy \\
\hline [36] & Effects of ultrasonic vibration on solidification structure and properties of $\mathrm{Mg}-8 \mathrm{Li}-3 \mathrm{Al}$ alloy \\
\hline [37] & Effect of ultrasonic power on grain refinement and purification processing of AZ80 alloy by ultrasonic treatment \\
\hline [38] & Effect of Ultrasonic Treatment in the Static and Dynamic Mechanical Behavior of AZ91D Mg Alloy \\
\hline [39] & Ultrasonic Effects on Microstructure Evolution and Mechanical Properties of AZ80 Magnesium Alloy \\
\hline [40] & $\begin{array}{l}\text { Effect of ultrasonic frequency on cavitation behavior, microstructure and mechanical properties of AZ80 } \\
\text { magnesium alloy }\end{array}$ \\
\hline
\end{tabular}

There are essentially two different approaches regarding the ultrasonic treatment for grain refinement: the direct application of the ultrasonic vibration to the melt isothermally or continuously during solidification. The articles discussed herein were categorized according to the route followed by their authors for ultrasonic treatment. Concerning this topic, there is no clear preference for one of these methods over the other since 8/17 works report results of ultrasonic melt treatment, and 9/17 correspond to ultrasonic vibration application during the material solidification.

The data gathered from each work is presented in Tables 2-5, summarizing the treatment parameters and the microstructural and mechanical characterization results, respectively. Table 6 reports the mechanisms that the authors suggest underlying the ultrasound's ability to produce finer microstructures, and Table 7 reviews the key findings of each work considered. 
Table 2. Treatment conditions used in studies of continuous application of ultrasonic treatment during material solidification.

\begin{tabular}{|c|c|c|c|c|c|c|c|c|c|c|c|c|c|}
\hline Reference & Alloy & $\begin{array}{l}\text { Mass } \\
\text { (g) }\end{array}$ & $\begin{array}{l}\text { Protective } \\
\text { Atmosphere }\end{array}$ & $\begin{array}{l}\text { US Power } \\
\text { (W) }\end{array}$ & $\underset{(\mu \mathrm{m})}{\text { Amplitude }}$ & $\begin{array}{c}\text { US } \\
\text { Frequency } \\
\text { (Hz) }\end{array}$ & $\begin{array}{c}\text { US } \\
\text { Temperature } \\
\left({ }^{\circ} \mathrm{C}\right)\end{array}$ & $\begin{array}{c}\text { US } \\
\text { Duration } \\
\text { (s) }\end{array}$ & $\begin{array}{c}\text { Pouring } \\
\text { Temperature } \\
\left({ }^{\circ} \mathrm{C}\right)\end{array}$ & $\begin{array}{l}\text { Crucible } \\
\text { Material }\end{array}$ & $\begin{array}{c}\text { Mold } \\
\text { Material }\end{array}$ & $\begin{array}{l}\text { Mold Tem- } \\
\text { perature } \\
\left({ }^{\circ} \mathrm{C}\right)\end{array}$ & OBS. \\
\hline [6] & $\begin{array}{l}\text { AZ31; } \\
\text { AZ80; } \\
\text { AZ91 }\end{array}$ & $\mathrm{NA}^{*}$ & $\mathrm{NA}^{*}$ & NA * & $25 \mu \mathrm{m}$ & 20 & From 725-750 & $\begin{array}{l}420 \text { (till near } \\
\text { the end of } \\
\text { solidifica- } \\
\text { tion) }\end{array}$ & $\begin{array}{l}\text { The melt } \\
\text { solidified } \\
\text { inside the } \\
\text { crucible. }\end{array}$ & $\begin{array}{l}\text { Clay } \\
\text { graphite }\end{array}$ & NA & NA & $\begin{array}{l}\text { (a) The melt was } \\
\text { homogenized at } \\
725-7500^{\circ} \mathrm{C} \text { after what } \\
\text { it was ultrasonicated. }\end{array}$ \\
\hline [10] & AZ91 & 150 & $\begin{array}{l}\text { Gas mixture } \\
\text { of } \mathrm{CO}_{2} / \mathrm{SF}_{6}\end{array}$ & NA * & NA * & 19 & $\begin{array}{l}\text { From } 615 \text { to } \\
\text { 580; From } 615 \\
\text { to } 595 ; \text { From } \\
595 \text { to } 590\end{array}$ & $\mathrm{NA}^{*}$ & $\begin{array}{l}\text { The melt } \\
\text { solidified } \\
\text { inside the } \\
\text { crucible. }\end{array}$ & Alumina & NA & NA & $\begin{array}{l}\text { (a) Before the UST, the } \\
\text { melt was superheated } \\
\text { up to } 720^{\circ} \mathrm{C} \text { and the } \\
\text { furnace was turned off. } \\
\text { (b) A thermocouple } \\
\text { was inserted near the } \\
\text { middle of the melt to } \\
\text { acquire the } \\
\text { temperature during } \\
\text { solidification. }\end{array}$ \\
\hline [28] & AZ31 & 350 & $\mathrm{NA}^{*}$ & $\begin{array}{c}1700 \\
\mathrm{~W} / \mathrm{cm}^{2}\end{array}$ & 30 & 20 & $\begin{array}{c}\text { From } 730 \text { to } \\
\quad 680\end{array}$ & 180 & $\begin{array}{l}\text { The melt } \\
\text { solidified } \\
\text { inside the } \\
\text { crucible. }\end{array}$ & $\begin{array}{l}\text { Boron } \\
\text { nitride- } \\
\text { coated } \\
\text { clay- } \\
\text { graphite }\end{array}$ & NA & NA & $\begin{array}{l}\text { (a) The material was } \\
\text { melted at } 730^{\circ} \mathrm{C} \text { after } \\
\text { what it was } \\
\text { withdrawn from the } \\
\text { furnace for } \\
\text { ultrasonication as the } \\
\text { melt reached } 680^{\circ} \mathrm{C} \text {. }\end{array}$ \\
\hline$[31]$ & AZ91D & 150 & $\begin{array}{l}\text { Gas mixture } \\
\text { of } \mathrm{CO}_{2} / \mathrm{SF}_{6}\end{array}$ & $\mathrm{NA}^{*}$ & NA * & 19 & $\begin{array}{c}\text { From } 615 \text { to } \\
580\end{array}$ & $\mathrm{NA}^{*}$ & $\begin{array}{l}\text { The melt } \\
\text { solidified } \\
\text { inside the } \\
\text { crucible. }\end{array}$ & Alumina & NA & NA & $\begin{array}{l}\text { (a) Before the UST, the } \\
\text { melt was superheated } \\
\text { up to } 720^{\circ} \mathrm{C} \text { and the } \\
\text { furnace was turned off. } \\
\text { (b) A thermocouple } \\
\text { was inserted near the } \\
\text { middle of the melt to } \\
\text { acquire the } \\
\text { temperature during } \\
\text { solidification. }\end{array}$ \\
\hline [32] & $\begin{array}{l}\text { AZ31; } \\
\text { AJ62; } \\
\text { AZ91 }\end{array}$ & 350 & $\mathrm{NA}^{*}$ & $\begin{array}{c}1400 \\
\mathrm{~W} / \mathrm{cm}^{2}\end{array}$ & 30 & 20 & From 680 & 180 & $\begin{array}{l}\text { The melt } \\
\text { solidified } \\
\text { inside the } \\
\text { crucible. }\end{array}$ & $\begin{array}{l}\text { Coated clay- } \\
\text { graphite }\end{array}$ & NA & NA & $\begin{array}{l}\text { (a) UT was applied at } \\
680^{\circ} \mathrm{C} \text { for } 180 \mathrm{~s} \text { after } \\
\text { the crucible had been } \\
\text { withdrawn from the } \\
\text { furnace. }\end{array}$ \\
\hline
\end{tabular}


Table 2. Cont

\begin{tabular}{|c|c|c|c|c|c|c|c|c|c|c|c|c|c|}
\hline Reference & Alloy & $\begin{array}{l}\text { Mass } \\
(\mathrm{g})\end{array}$ & $\begin{array}{l}\text { Protective } \\
\text { Atmosphere }\end{array}$ & $\begin{array}{l}\text { US Power } \\
\text { (W) }\end{array}$ & $\begin{array}{c}\text { Amplitude } \\
(\mu \mathrm{m})\end{array}$ & $\begin{array}{c}\text { US } \\
\text { Frequency } \\
\text { (Hz) }\end{array}$ & $\begin{array}{c}\text { US } \\
\text { Temperature } \\
\left({ }^{\circ} \mathrm{C}\right)\end{array}$ & $\begin{array}{c}\text { US } \\
\text { Duration } \\
\text { (s) }\end{array}$ & $\begin{array}{c}\text { Pouring } \\
\text { Temperature } \\
\left({ }^{\circ} \mathrm{C}\right)\end{array}$ & $\begin{array}{l}\text { Crucible } \\
\text { Material }\end{array}$ & $\begin{array}{c}\text { Mold } \\
\text { Material }\end{array}$ & $\begin{array}{l}\text { Mold Tem- } \\
\text { perature } \\
\left({ }^{\circ} \mathrm{C}\right)\end{array}$ & OBS. \\
\hline [33] & AZ91 & NA* & $\begin{array}{l}\text { Gas mixture } \\
\text { of } \mathrm{CO}_{2}-2 \% \mathrm{SF}_{6}\end{array}$ & $\begin{array}{l}300 ; 500 \\
\quad 700\end{array}$ & NA * & 20 & From 730 & NA* & 730 & NA* & Sand & $\mathrm{NA}^{*}$ & $\begin{array}{l}\text { (a) The melt was } \\
\text { poured at } 730^{\circ} \mathrm{C} \text { to a } \\
\text { sand mold after which } \\
\text { it was ultrasonicated. }\end{array}$ \\
\hline [36] & $\begin{array}{l}\mathrm{Mg}-8 \mathrm{Li}- \\
3 \mathrm{Al}\end{array}$ & NA* & $\begin{array}{c}\text { Ar } \\
\text { atmosphere }+ \\
\text { molten flux of } \\
75 \% \mathrm{LiCl}+ \\
25 \% \mathrm{LiF}\end{array}$ & $\begin{array}{c}50 ; 110 ; \\
170 ; 210 \\
260\end{array}$ & NA* & 20 & NA * & 90 & $\mathrm{NA}^{*}$ & Steel & $\begin{array}{l}\text { Stainless } \\
\text { Steel }\end{array}$ & 600 & $\begin{array}{l}\text { (a) The melt was } \\
\text { poured to a } \\
\text { stainless-steel mold } \\
\text { preheated up to } \\
600^{\circ} \mathrm{C} \text {, in a preserving } \\
\text { heat furnace. }\end{array}$ \\
\hline [38] & AZ91D & NA * & $\begin{array}{l}\text { Gas mixture } \\
\text { of } \mathrm{SF}_{6} / \mathrm{CO}_{2}\end{array}$ & 400 & NA * & 20.1 & $\begin{array}{l}\text { From } 680 \text { to } \\
\quad 608\end{array}$ & NA * & 680 & NA* & $\begin{array}{l}\text { Stainless } \\
\text { Steel }\end{array}$ & $\mathrm{NA}^{*}$ & $\begin{array}{l}\text { (a) The melt was kept } \\
\text { at } 700^{\circ} \mathrm{C} \text { for } 15 \mathrm{~min} \\
\text { for homogenization. } \\
\text { The molten alloy was } \\
\text { then allowed to cool to } \\
680^{\circ} \mathrm{C} \text { before pouring. }\end{array}$ \\
\hline [39] & AZ80 & 750 & $\begin{array}{c}\text { Gas mixture } \\
\text { of } \mathrm{CO}_{2}+0.5 \% \\
\mathrm{SF}_{6}\end{array}$ & 600 & NA* & 20 & $\begin{array}{l}\text { From } 650 \text { to } \\
\quad 571\end{array}$ & NA* & $\begin{array}{l}\text { The melt } \\
\text { solidified } \\
\text { inside the } \\
\text { crucible. }\end{array}$ & $\begin{array}{l}\text { Stainless } \\
\text { steel }\end{array}$ & NA & NA & $\begin{array}{l}\text { (a) The alloy was } \\
\text { heated up to } 720^{\circ} \mathrm{C} \\
\text { and kept at that } \\
\text { temperature for } \\
15 \text { min for } \\
\text { homogenization after } \\
\text { what the furnace was } \\
\text { turned off. (b) After } \\
\text { ultrasonication, the } \\
\text { crucible was } \\
\text { withdrawn from the } \\
\text { furnace. }\end{array}$ \\
\hline
\end{tabular}


Table 3. Treatment conditions used in studies of isothermal application of ultrasonic treatment to the melt.

\begin{tabular}{|c|c|c|c|c|c|c|c|c|c|c|c|c|c|}
\hline Reference & Alloy & $\begin{array}{c}\text { Mass } \\
\text { (g) }\end{array}$ & $\begin{array}{l}\text { Protective } \\
\text { Atmosphere }\end{array}$ & $\begin{array}{l}\text { US Power } \\
\text { (W) }\end{array}$ & $\underset{(\mu \mathrm{m})}{\text { Amplitude }}$ & $\begin{array}{c}\text { US } \\
\text { Frequency } \\
\text { (Hz) }\end{array}$ & $\begin{array}{l}\text { US Temper- } \\
\text { ature } \\
\left({ }^{\circ} \mathrm{C}\right)\end{array}$ & $\begin{array}{c}\text { US } \\
\text { Duration } \\
\text { (s) }\end{array}$ & $\begin{array}{c}\text { Pouring } \\
\text { Temperature } \\
\left({ }^{\circ} \mathrm{C}\right)\end{array}$ & $\begin{array}{l}\text { Crucible } \\
\text { Material }\end{array}$ & $\begin{array}{l}\text { Mould } \\
\text { Material }\end{array}$ & $\begin{array}{c}\text { Mould } \\
\text { Temperature } \\
\left({ }^{\circ} \mathrm{C}\right)\end{array}$ & OBS. \\
\hline [27] & AZ91 & $\mathrm{NA}^{*}$ & $\begin{array}{l}\text { Gas mixture } \\
\text { of } \mathrm{CO}_{2} / \mathrm{SF}_{6}\end{array}$ & $\begin{array}{l}400,600 \\
800,1000\end{array}$ & $\mathrm{NA}^{*}$ & 20 & 680 & 100 & $\begin{array}{l}\text { The melt } \\
\text { solidified inside } \\
\text { the crucible }\end{array}$ & $\begin{array}{l}\text { Stainless } \\
\text { Steel }\end{array}$ & $\begin{array}{l}\text { Metal (not } \\
\text { specified) }\end{array}$ & $\mathrm{NA}^{*}$ & $\begin{array}{l}\text { (a) The melt was } \\
\text { modified with } \mathrm{C}_{2} \mathrm{Cl}_{6} \\
\text { at } 745^{\circ} \mathrm{C} \text { and } \\
\text { purified with } \mathrm{Ar}_{2} \text { at } \\
720^{\circ} \mathrm{C} \text {, before the } \\
\text { ultrasonication. (b) } \\
\text { A thermocouple was } \\
\text { inserted near the } \\
\text { middle of the melt } \\
\text { to acquire the } \\
\text { temperature during } \\
\text { solidification. }\end{array}$ \\
\hline [3] & AZ91E & 1000 & $\begin{array}{c}\text { Gas mixture } \\
\text { of } \mathrm{CO}_{2}-0.5 \% \\
\mathrm{SF}_{6}\end{array}$ & $\mathrm{NA}^{*}$ & $30 \mu \mathrm{m}$ & 20 & 740 & $\begin{array}{r}60 ; 120 \\
180 ; 240\end{array}$ & 720 & $\begin{array}{l}\text { Low } \\
\text { carbon } \\
\text { steel }\end{array}$ & Steel & 350 & - \\
\hline [8] & AZ91 & 320 & $\begin{array}{l}\text { MAGREX flux } \\
\text { (Foseco } \\
\text { Foundry } \\
\text { International } \\
\text { Limited) }\end{array}$ & $120,240,360$ & $\mathrm{NA}^{*}$ & 20 & 700 & 300 & 700 & $\mathrm{NA}^{*}$ & Sand & $\mathrm{NA}^{*}$ & - \\
\hline [9] & AS41 & 200 & Ar flow & $\begin{array}{c}4300 \\
W / \mathrm{cm}^{2}\end{array}$ & $48 \mu \mathrm{m}$ & $\mathrm{NA}^{*}$ & $605 ; 620$ & $30 ; 60 ; 90$ & $605 ; 620$ & Mild steel & $\mathrm{NA}^{*}$ & $\mathrm{NA}^{*}$ & $\begin{array}{l}\text { (a) The material was } \\
\text { melted at } 700^{\circ} \mathrm{C}\end{array}$ \\
\hline [34] & $\begin{array}{l}\text { AM60; } \\
\text { AZ91 }\end{array}$ & 3000 & $\begin{array}{l}\text { Gas mixture } \\
\text { of } \mathrm{CO}_{2} / \mathrm{SF}_{6}\end{array}$ & $500 ; 1000$ & $\mathrm{NA}^{*}$ & $15 ; 20$ & 650 & $\begin{array}{c}180 ; 300 \\
600\end{array}$ & 650 & $\begin{array}{l}\text { Stainless } \\
\text { steel }\end{array}$ & $\begin{array}{l}\text { Water- } \\
\text { cooled } \\
\text { copper } \\
\text { mould }\end{array}$ & $\mathrm{NA}^{*}$ & - \\
\hline [35] & AZ91 & 1000 & $\begin{array}{l}\text { Gas mixture } \\
\text { of } \mathrm{CO}_{2} / \mathrm{SF}_{6}\end{array}$ & 350 & $\mathrm{NA}^{*}$ & 20 & 700 & 1200 & 720 & $\mathrm{NA}^{*}$ & Steel & 450 & $\begin{array}{l}\text { (a) The UST was } \\
\text { applied at } 700^{\circ} \mathrm{C} \\
\text { after what the melt } \\
\text { was heated up to } \\
720^{\circ} \mathrm{C}\end{array}$ \\
\hline
\end{tabular}


Table 3. Cont

\begin{tabular}{|c|c|c|c|c|c|c|c|c|c|c|c|c|c|}
\hline Reference & Alloy & $\begin{array}{l}\text { Mass } \\
\text { (g) }\end{array}$ & $\begin{array}{l}\text { Protective } \\
\text { Atmosphere }\end{array}$ & $\begin{array}{l}\text { US Power } \\
\text { (W) }\end{array}$ & $\underset{(\mu \mathrm{m})}{\text { Amplitude }}$ & $\begin{array}{c}\text { US } \\
\text { Frequency } \\
\text { (Hz) }\end{array}$ & $\begin{array}{l}\text { US Temper- } \\
\text { ature } \\
\left({ }^{\circ} \mathrm{C}\right)\end{array}$ & $\begin{array}{c}\text { US } \\
\text { Duration } \\
\text { (s) }\end{array}$ & $\begin{array}{c}\text { Pouring } \\
\text { Temperature } \\
\left({ }^{\circ} \mathrm{C}\right)\end{array}$ & $\begin{array}{l}\text { Crucible } \\
\text { Material }\end{array}$ & $\begin{array}{l}\text { Mould } \\
\text { Material }\end{array}$ & $\begin{array}{c}\text { Mould } \\
\text { Temperature } \\
\left({ }^{\circ} \mathrm{C}\right)\end{array}$ & OBS. \\
\hline [37] & AZ80 & 550 & $\begin{array}{c}\text { Gas mixture } \\
\text { of } \mathrm{CO}_{2}-0.5 \% \\
\mathrm{SF}_{6}\end{array}$ & $\begin{array}{r}230 ; 600 ; \\
950 ; 1400\end{array}$ & NA * & 20 & 650 & $\mathrm{NA}^{*}$ & $\begin{array}{l}\text { The melt } \\
\text { solidified inside } \\
\text { the crucible }\end{array}$ & Iron & NA & NA & $\begin{array}{l}\text { (a) The melt was } \\
\text { kept at } 650^{\circ} \mathrm{C} \text { for } \\
600 \mathrm{~s} \text { before the } \\
\text { ultrasonication. (b) } \\
\text { After } \\
\text { ultrasonication, the } \\
\text { melt was } \\
\text { water-quenched } \\
\text { immediatly. }\end{array}$ \\
\hline [40] & AZ80 & 5000 & $\begin{array}{c}\text { Gas mixture } \\
\text { of } \mathrm{CO}_{2}-0.5 \% \\
\mathrm{SF}_{6}\end{array}$ & $\begin{array}{c}1200 \\
\text { (Single); } 600 \\
+600 \text { (Dual) }\end{array}$ & $\mathrm{NA}^{*}$ & $\begin{array}{l}15 ; 20 \\
15+20\end{array}$ & 650 & 300 & 650 & Iron & $\begin{array}{l}\text { Water- } \\
\text { cooled } \\
\text { copper } \\
\text { mould }\end{array}$ & NA * & - \\
\hline
\end{tabular}

NA *-Not Available; NA—Not Applicable.

Table 4. Summary of microstructural analysis results reported in the included articles.

\begin{tabular}{|c|c|c|c|c|c|c|c|c|}
\hline \multirow{3}{*}{ Reference } & \multirow{3}{*}{ Type } & \multicolumn{7}{|c|}{ Microstructural Analysis } \\
\hline & & \multicolumn{2}{|r|}{ Phase Composition/Morphology } & \multicolumn{2}{|c|}{ Grain Size $(\mu \mathrm{m})$} & \multicolumn{2}{|c|}{ Sphericity } & \multirow{2}{*}{$\begin{array}{l}\text { Characterization } \\
\text { Methods }\end{array}$} \\
\hline & & AC & UST & $\mathrm{AC}$ & UST & $\mathrm{AC}$ & UST & \\
\hline [27] & $\begin{array}{l}\text { Isothermal } \\
\text { Treatment }\end{array}$ & $\begin{array}{l}\text { Equiaxed grains of } \alpha-\mathrm{Mg} \\
\text { phase with dot-like } \\
\text { particles distributed in the } \\
\text { grains. }\end{array}$ & $\begin{array}{l}\text { No significant difference concerning the } \alpha-\mathrm{Mg} \text { phase was } \\
\text { noticed between the untreated and ultrasonicated samples. } \\
\text { With the increase of the applied ultrasonic power, the phase } \\
\text { composition remained similar, and the size, fraction and } \\
\text { distribution of the intermetallic phases changed remarkably. } \\
\text { Eutectic phase: the area percentage of lamellar eutectic } \\
\text { phase increased to the maximum when the applied } \\
\text { ultrasonic power was } 600 \mathrm{~W} \text {. Further increase of ultrasonic } \\
\text { power led to the gradual decrease of the area percentage. } \\
\mathrm{Mg}_{17} \mathrm{Al}_{12} \text { : the area percentage of } \mathrm{Mg}_{17} \mathrm{Al}_{12} \text { decreased as the } \\
\text { ultrasonic power increased. } \mathrm{Al}_{8} \mathrm{Mn}_{5}: \text { the average area of } \\
\mathrm{Al}_{8} \mathrm{Mn} \mathrm{Mn}_{5} \text { particles reduced as the ultrasonic power rose up to } \\
600 \mathrm{~W} \text {, increasing rapidly when the ultrasonic power was } \\
\text { increased up to } 1000 \mathrm{~W} \text {. }\end{array}$ & & NA * & & & $\mathrm{OM}+\mathrm{SEM}$ \\
\hline
\end{tabular}


Table 4. Cont.

\begin{tabular}{|c|c|c|c|c|c|c|c|c|}
\hline \multirow{3}{*}{ Reference } & \multirow{3}{*}{ Type } & \multicolumn{7}{|c|}{ Microstructural Analysis } \\
\hline & & \multicolumn{2}{|r|}{ Phase Composition/Morphology } & \multicolumn{2}{|c|}{ Grain Size $(\mu \mathrm{m})$} & \multicolumn{2}{|c|}{ Sphericity } & \multirow{2}{*}{$\begin{array}{l}\text { Characterization } \\
\text { Methods }\end{array}$} \\
\hline & & $\mathrm{AC}$ & UST & AC & UST & AC & UST & \\
\hline [3] & $\begin{array}{l}\text { Isothermal } \\
\text { Treatment }\end{array}$ & $\begin{array}{l}\alpha-\mathrm{Mg} \text { phase: } \\
\text { coarse-grained structure } \\
\text { with a combination of } \\
\text { equiaxed and elongated } \\
\text { grains. } \mathrm{Mg}_{17} \mathrm{Al}_{12} \text { : large } \\
\text { continuous network } \\
\text { precipitated along grain } \\
\text { boundaries. Mn-Al: blocky } \\
\text { and needle-like shapes not } \\
\text { homogeneously } \\
\text { distributed through the } \\
\text { matrix since large clusters } \\
\text { could be observed. }\end{array}$ & $\begin{array}{l}\alpha \text {-Mg phase: the grain size was finer than the untreated } \\
\text { sample when sonicated for } 60 \mathrm{~s} \text {. However, the } \\
\text { microstructure still presented a semi-equiaxed structure } \\
\text { composed of elongated and equiaxed grain. Additional } \\
\text { sonication to } 120 \mathrm{~s} \text { improved the grain size and morphology } \\
\text { even further and the microstructure evolved to a more } \\
\text { equiaxed state with a reduced number of elongated grains. } \\
\text { Upon sonicating for } 180 \mathrm{~s} \text {, the microstructure became } \\
\text { completely equiaxed with a significant decrease of the grain } \\
\text { size. Increasing the ultrasonic treatment time from } 180 \text { to } 240 \\
\text { s did not significantly influence the grain size and } \\
\text { morphology. Intermetallic phase: as the sonication time } \\
\text { increased, the } \mathrm{Mg}_{17} \mathrm{Al}_{12} \text { networks became more refined. The } \\
\text { ultrasonication treatment effectively reduced the overall } \\
\text { length of the eutectic networks and improved their } \\
\text { distribution. Upon sonicating the alloy for } 120 \mathrm{~s} \text {, the Mn-Al } \\
\text { intermetallics became more homogeneously distributed } \\
\text { throughout the alloy matrix with minimal signs of } \\
\text { agglomeration. }\end{array}$ & 202 & $\begin{array}{l}144(60 \mathrm{~s}) ; 109(120 \mathrm{~s}) \\
50(180 \mathrm{~s}, 240 \mathrm{~s})\end{array}$ & NA* & $\mathrm{NA}^{*}$ & $\mathrm{OM}+\mathrm{SEM}$ \\
\hline [6] & $\begin{array}{c}\text { Continuous } \\
\text { Treatment }\end{array}$ & $\begin{array}{l}\text { Dendritic structure with } \\
\text { long arms of } \alpha \text {-Mg phase. }\end{array}$ & $\begin{array}{l}\text { Non-dendritic finer grains of } \alpha-\mathrm{Mg} \text { phase. A gradual } \\
\text { increase in the grain size was observed with increasing } \\
\text { distance from the ultrasound radiator. }\end{array}$ & & NA * & & & $\mathrm{OM}$ \\
\hline
\end{tabular}


Table 4. Cont.

\begin{tabular}{|c|c|c|c|c|c|c|c|c|}
\hline \multirow{3}{*}{ Reference } & \multirow{3}{*}{ Type } & \multicolumn{7}{|c|}{ Microstructural Analysis } \\
\hline & & \multicolumn{2}{|r|}{ Phase Composition/Morphology } & \multicolumn{2}{|c|}{ Grain Size $(\mu \mathrm{m})$} & \multicolumn{2}{|c|}{ Sphericity } & \multirow{2}{*}{$\begin{array}{l}\text { Characterization } \\
\text { Methods }\end{array}$} \\
\hline & & AC & UST & AC & UST & AC & UST & \\
\hline [8] & $\begin{array}{l}\text { Isothermal } \\
\text { Treatment }\end{array}$ & $\begin{array}{l}\alpha \text {-Mg phase: coarse and } \\
\text { non-uniform dendritic } \\
\text { microstructure. } \\
\mathrm{Mg}_{17} \mathrm{Al}_{12} \text { : continuous } \\
\text { network at the grain } \\
\text { boundaries. } \mathrm{Mg}_{2} \mathrm{Si}: \\
\text { angular particles dispersed } \\
\text { in the matrix. MgFeAl(Si): } \\
\text { relatively spherical phase } \\
\text { dispersed in the matrix. }\end{array}$ & $\begin{array}{l}\alpha \text {-Mg phase: finer and equiaxed dendrites. The grain size } \\
\text { decreased, and the grain uniformity increased as the } \\
\text { ultrasound power increased. Although the grains were still } \\
\text { dendritic, they exhibited fewer branches and shorter arms at } \\
\text { higher ultrasonic power, leading to higher sphericity. } \\
\mathrm{Mg}_{17} \mathrm{Al}_{12} \text { : smaller and more fragmented particles. The } \\
\text { sphericity of the particles, their uniformity and distribution } \\
\text { were enhanced. Higher ultrasonic power led to better results. } \\
\mathrm{Mg}_{2} \mathrm{Si} \text { : globular and finer particles. The results revealed a } \\
\text { decreasing trend for } \mathrm{Mg}_{2} \mathrm{Si} \text { particle size and an increasing } \\
\text { trend for their sphericity by increasing the ultrasonic power. } \\
\text { Application of } 360 \mathrm{~W} \text { has produced the most refined } \\
\text { particles with all } \mathrm{Mg}_{2} \mathrm{Si} \text { particles. } \mathrm{MgFeAl}(\mathrm{Si}) \text { : smaller and } \\
\text { rounder particles. The increase of ultrasonic power led to } \\
\text { better results, however, since these particles are intrinsically } \\
\text { round, the effect of ultrasonic treatment was not as } \\
\text { significant as on the other intermetallic phases. }\end{array}$ & 280 & $\begin{array}{c}180(120 \mathrm{~W}, 240 \mathrm{~W}) \\
125(360 \mathrm{~W})\end{array}$ & 0.3 & $\begin{array}{c}0.375 \\
(120 \mathrm{~W}) \\
0.425 \\
(240 \mathrm{~W}) \\
0.45 \\
(360 \mathrm{~W})\end{array}$ & $\mathrm{OM}+\mathrm{SEM}$ \\
\hline [9] & $\begin{array}{l}\text { Isothermal } \\
\text { Treatment }\end{array}$ & $\begin{array}{l}\text { Coarse dendrites with long } \\
\text { arms of } \alpha \text {-Mg throughout } \\
\text { the samples, } \\
\text { demonstrating the dendrite } \\
\text { growth mode. }\end{array}$ & $\begin{array}{l}\text { Equiaxed grains of primary } \alpha-\mathrm{Mg} \text { phase were found along } \\
\text { with a network of } \mathrm{Mg}_{17} \mathrm{Al}_{12} \text { intermetallic phase. } \mathrm{Mg}_{2} \mathrm{Si} \\
\text { phase in Chinese script structure segregated along the grain } \\
\text { boundary. The microstructure obtained after ultrasonication } \\
\text { at } 605^{\circ} \mathrm{C} \text { was finer and more globular than that of the } \\
\text { material ultrasonicated at } 620^{\circ} \mathrm{C} \text {. The increase of ultrasonic } \\
\text { treatment duration led to finer grains, regardless of the } \\
\text { processing temperature. }\end{array}$ & $\begin{array}{l}375(605 \\
\left.{ }^{\circ} \mathrm{C}\right) ; 330 \\
\left(620^{\circ} \mathrm{C}\right)\end{array}$ & $\begin{array}{c}605^{\circ} \mathrm{C}: \approx 175(30 \mathrm{~s}) ; \\
\approx 125(60 \mathrm{~s}) ; \approx 92 \\
(90 \mathrm{~s}) ; 620^{\circ} \mathrm{C}: \approx 240 \\
(30 \mathrm{~s}) ; \approx 147(60 \mathrm{~s}, \\
90 \mathrm{~s})\end{array}$ & $\mathrm{NA}^{*}$ & $\mathrm{NA}^{*}$ & $\mathrm{OM}+\mathrm{SEM}$ \\
\hline [10] & $\begin{array}{c}\text { Continuous } \\
\text { Treatment }\end{array}$ & $\begin{array}{l}\text { Coarse dendrites of } \alpha-\mathrm{Mg} \\
\text { phase throughout the } \\
\text { sample, demonstrating the } \\
\text { normal dendrite growth } \\
\text { mode. }\end{array}$ & $\begin{array}{l}\text { The temperature at which the ultrasonic vibration was } \\
\text { applied significantly changed the morphology and size of } \\
\qquad \alpha \text {-Mg phase. } \\
\text { From } 615^{\circ} \mathrm{C} \text { to } 595^{\circ} \mathrm{C} \\
\text { Fine uniform grains of } \alpha \text { - Mg } \\
\text { From } 595^{\circ} \mathrm{C} \text { to } 590^{\circ} \mathrm{C} \text { and from } 590^{\circ} \mathrm{C} \text { to } 580^{\circ} \mathrm{C} \\
\text { Dendritic grains of } \alpha \text { - Mg. The microstructure of US-treated } \\
\text { samples in the temperature range } 580-590^{\circ} \mathrm{C} \text { was coarser } \\
\text { than that of samples treated at } 5955^{\circ} \mathrm{C} \text { to } 5900^{\circ} \mathrm{C} \text {, but slightly } \\
\text { more refined than that without ultrasonic vibration. }\end{array}$ & 500 & NA * & $\mathrm{NA}^{*}$ & NA * & $\mathrm{OM}$ \\
\hline
\end{tabular}


Table 4. Cont.

\begin{tabular}{|c|c|c|c|c|c|c|c|c|}
\hline \multirow{3}{*}{ Reference } & \multirow{3}{*}{ Type } & \multicolumn{7}{|c|}{ Microstructural Analysis } \\
\hline & & \multicolumn{2}{|r|}{ Phase Composition/Morphology } & \multicolumn{2}{|c|}{ Grain Size $(\mu \mathrm{m})$} & \multicolumn{2}{|c|}{ Sphericity } & \multirow{2}{*}{$\begin{array}{l}\text { Characterization } \\
\text { Methods }\end{array}$} \\
\hline & & AC & UST & AC & UST & AC & UST & \\
\hline [28] & $\begin{array}{l}\text { Continuous } \\
\text { Treatment }\end{array}$ & $\begin{array}{l}\text { Coarse equiaxed dendritic } \\
\text { structure throughout the } \\
\text { ingot. }\end{array}$ & $\begin{array}{l}\text { Refined equiaxed grain structure only below the ultrasound } \\
\text { radiating face when the sonotrode was deeply immersed in } \\
\text { the melt. No noticeable refinement occurred adjacent to the } \\
\text { cylindrical face of the sonotrode near the melt surface. When } \\
\text { the sonotrode was positioned just touching the solidifying } \\
\text { melt, equiaxed grain structure was obtained at the central } \\
\text { region of the sample. }\end{array}$ & 2000 & $\begin{array}{l}107 \text { ( } 5 \mathrm{~mm} \text { below the } \\
\text { ultrasound radiating } \\
\text { face) }\end{array}$ & $\mathrm{NA}^{*}$ & NA * & Macroscopy \\
\hline [31] & $\begin{array}{l}\text { Continuous } \\
\text { Treatment }\end{array}$ & $\begin{array}{l}\text { Coarse dendrites of } \alpha-\mathrm{Mg} \\
\text { phase throughout the } \\
\text { samples, demonstrating } \\
\text { the normal dendrite } \\
\text { growth. }\end{array}$ & $\begin{array}{l}\text { Fine grains of } \alpha \text {-Mg phase. The grain morphology is } \\
\text { significantly different since it was modified from developed } \\
\text { dendrites to non-dendrites grains. }\end{array}$ & 900 & 195 & $\mathrm{NA}^{*}$ & NA * & OM+EPMA \\
\hline [32] & $\begin{array}{l}\text { Continuous } \\
\text { Treatment }\end{array}$ & NA * & Finer grains of $\alpha-\mathrm{Mg}$ & NA * & NA * & $\mathrm{NA} *$ & $\mathrm{NA}^{*}$ & $\mathrm{OM}$ \\
\hline [33] & $\begin{array}{l}\text { Continuous } \\
\text { Treatment }\end{array}$ & $\begin{array}{l}\text { Coarse dendrites of } \alpha-\mathrm{Mg} \\
\text { throughout the samples, } \\
\text { demonstrating the dendrite } \\
\text { growth mode. }\end{array}$ & $\begin{array}{l}\text { The dendritic structure was fragmented, and a more } \\
\text { globular phase formed. A gradual decrease of the grain size } \\
\text { was noticed as the ultrasonic power increased. Although the } \\
\text { grain size decreased slightly at } 300 \mathrm{~W} \text { of ultrasonic power, } \\
\text { the grain morphology was still coarse. When the ultrasonic } \\
\text { power increased up to } 700 \mathrm{~W} \text {, the } \alpha \text {-Mg grains became finer } \\
\text { and globular. }\end{array}$ & 202 & $\begin{array}{c}195(300 \mathrm{~W}) ; 152 \\
(500 \mathrm{~W}) ; 146(700 \mathrm{~W})\end{array}$ & $\mathrm{NA}^{*}$ & NA * & $\mathrm{OM}$ \\
\hline [34] & $\begin{array}{l}\text { Isothermal } \\
\text { Treatment }\end{array}$ & $\begin{array}{l}\text { Large dendritic grains of } \\
\alpha-\mathrm{Mg} \text { phase. }\end{array}$ & $\begin{array}{l}\text { Finer and more homogeneous microstructure. Tiny particles } \\
\text { from the melt were noticed dispersed in } \mathrm{Mg}_{17} \mathrm{Al}_{12} \text { phase. } \\
\text { When the frequency increased from } 15 \mathrm{kHz} \text { to } 20 \mathrm{kHz} \text {, the } \\
\text { grains became finer. }\end{array}$ & $\begin{array}{l}\text { AZ91: 205; } \\
\text { AM60: } 200\end{array}$ & $\begin{array}{l}\text { AZ91: } 125(600 \mathrm{~s}, \\
20 \mathrm{kHz}) ; \text { AM60: } 90 \\
(600 \mathrm{~s}, 20 \mathrm{kHz})\end{array}$ & $\mathrm{NA}^{*}$ & NA * & $\mathrm{OM}+\mathrm{SEM}$ \\
\hline [35] & $\begin{array}{l}\text { Isothermal } \\
\text { Treatment }\end{array}$ & $\begin{array}{l}\text { Microstructure composed } \\
\text { of primary } \alpha-\mathrm{Mg}_{\text {and }} \\
\text { eutectic phase } \mathrm{Mg}_{17} \mathrm{Al}_{12} \text {. } \\
\mathrm{Mg}_{17} \mathrm{Al}_{12} \text { : plates located } \\
\text { mainly at grain boundaries. }\end{array}$ & $\begin{array}{l}\alpha-\mathrm{Mg} \text { phase: no relevant changes were noticed. } \mathrm{Mg}_{17} \mathrm{Al}_{12} \text { : } \\
\text { finer and oriented lamellar phase along the grain } \\
\text { boundaries. }\end{array}$ & & NA * & & & $\mathrm{OM}+\mathrm{SEM}$ \\
\hline
\end{tabular}


Table 4. Cont.

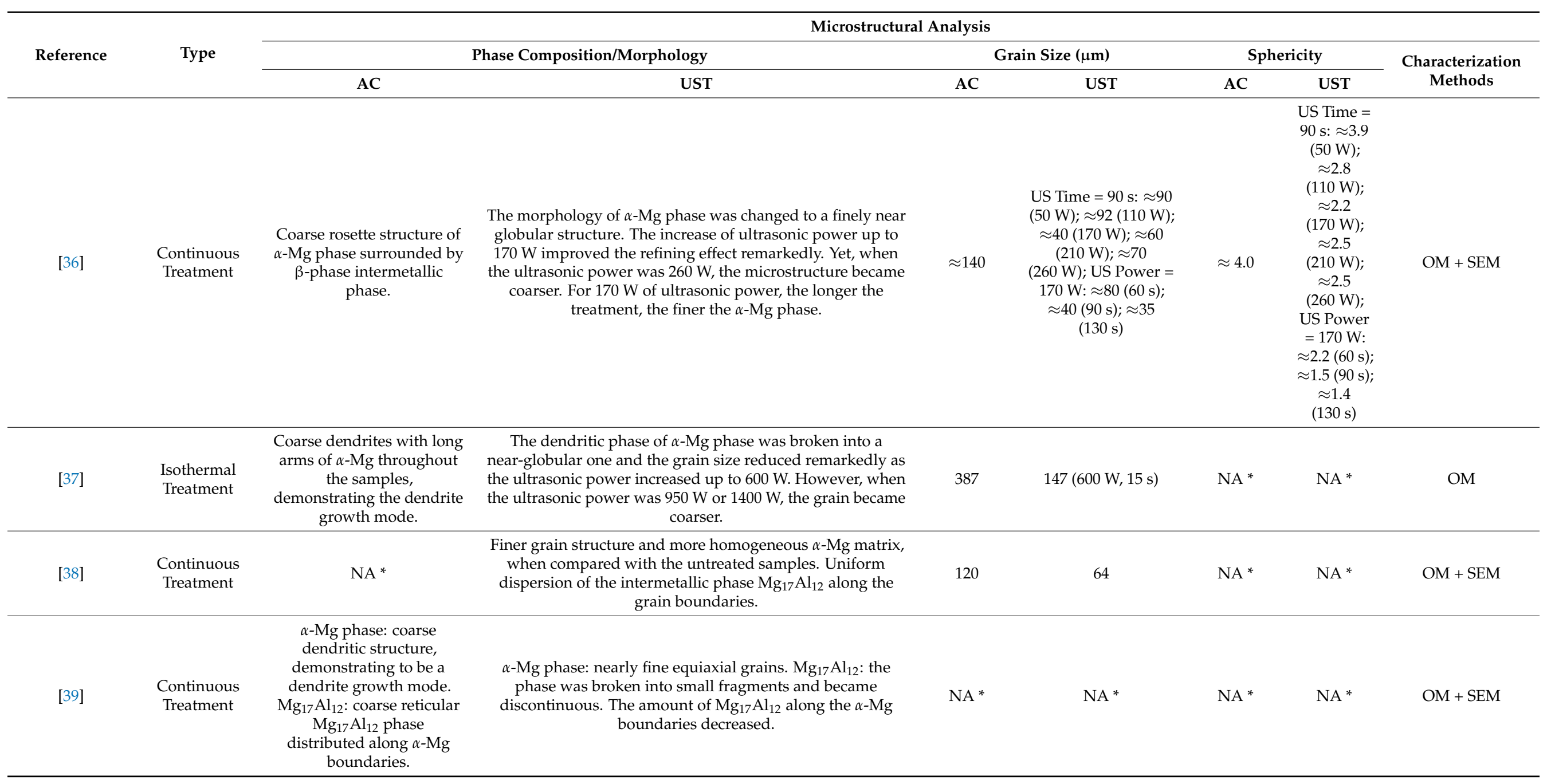


Table 4. Cont.

\begin{tabular}{|c|c|c|c|c|c|c|c|c|}
\hline \multirow{3}{*}{ Reference } & \multirow{3}{*}{ Type } & \multicolumn{7}{|c|}{ Microstructural Analysis } \\
\hline & & \multicolumn{3}{|c|}{ Phase Composition/Morphology } & \multicolumn{2}{|c|}{ Grain Size $(\mu \mathrm{m})$} & Sphericity & \multirow{2}{*}{$\begin{array}{l}\text { Characterization } \\
\text { Methods }\end{array}$} \\
\hline & & \multicolumn{3}{|c|}{ UST } & AC & UST & AC & \\
\hline [40] & $\begin{array}{l}\text { Isothermal } \\
\text { Treatment }\end{array}$ & $\begin{array}{l}\alpha-\mathrm{Mg} \text { phase: dendritic } \\
\text { microstructure with } \\
\text { developed secondary } \\
\text { dendrite arms. } \mathrm{Mg}_{17} \mathrm{Al}_{12}: \\
\text { continuous network along } \\
\text { the grain boundaries. }\end{array}$ & $\begin{array}{l}\text { SUT } 20 \mathrm{kHz} \text { : the grains } \\
\text { dendrite arms were short } \\
\text { phase was fragmented, a } \\
15 \mathrm{kHz} \text { : the secondary } \\
\text { and the roundness was ir } \\
\text { point-like second phase } \\
\text { finer and spherical. The } \\
\text { more dispersed and exh } \\
\text { strip. The intermetallic }\end{array}$ & $\begin{array}{l}\text { nd the secondary } \\
\text { y. The intermetallic } \\
\text { rea decreased. SUT } \\
\text { nost disappeared, } \\
\text { ificant amount of a } \\
\text { the grains became } \\
\text { second phase was } \\
\text { of point and short } \\
\text { ea decreases to its }\end{array}$ & \multicolumn{2}{|r|}{$\begin{array}{c}123(\text { SUT } 20 \mathrm{kHz}) ; 99 \\
\text { (SUT } 15 \mathrm{kHz}) ; 80 \\
\text { (DUT) }\end{array}$} & $\mathrm{NA}^{*}$ & OM + SEM \\
\hline \multirow{2}{*}{ Reference } & \multirow{2}{*}{ Type } & \multicolumn{7}{|c|}{ Mechanical Properties } \\
\hline & & AC & UST & UST & $\mathrm{AC}$ & UST & AC & UST \\
\hline [27] & $\begin{array}{l}\text { Isothermal } \\
\text { Treatment }\end{array}$ & \multicolumn{7}{|c|}{ NA * } \\
\hline [3] & $\begin{array}{l}\text { Isothermal } \\
\text { Treatment }\end{array}$ & 95 & 138 & 161 & $1.35 \%$ & $2.2 \%$ & $\mathrm{NA}^{*}$ & NA * \\
\hline [6] & $\begin{array}{c}\text { Continuous } \\
\text { Treatment }\end{array}$ & \multicolumn{7}{|c|}{ NA * } \\
\hline [8] & $\begin{array}{l}\text { Isothermal } \\
\text { Treatment }\end{array}$ & \multicolumn{7}{|c|}{$\mathrm{NA} *$} \\
\hline [9] & $\begin{array}{l}\text { Isothermal } \\
\text { Treatment }\end{array}$ & \multicolumn{7}{|c|}{$\mathrm{NA} *$} \\
\hline [10] & $\begin{array}{c}\text { Continuous } \\
\text { Treatment }\end{array}$ & \multicolumn{7}{|c|}{$\mathrm{NA}^{*}$} \\
\hline
\end{tabular}


Table 5. Cont.

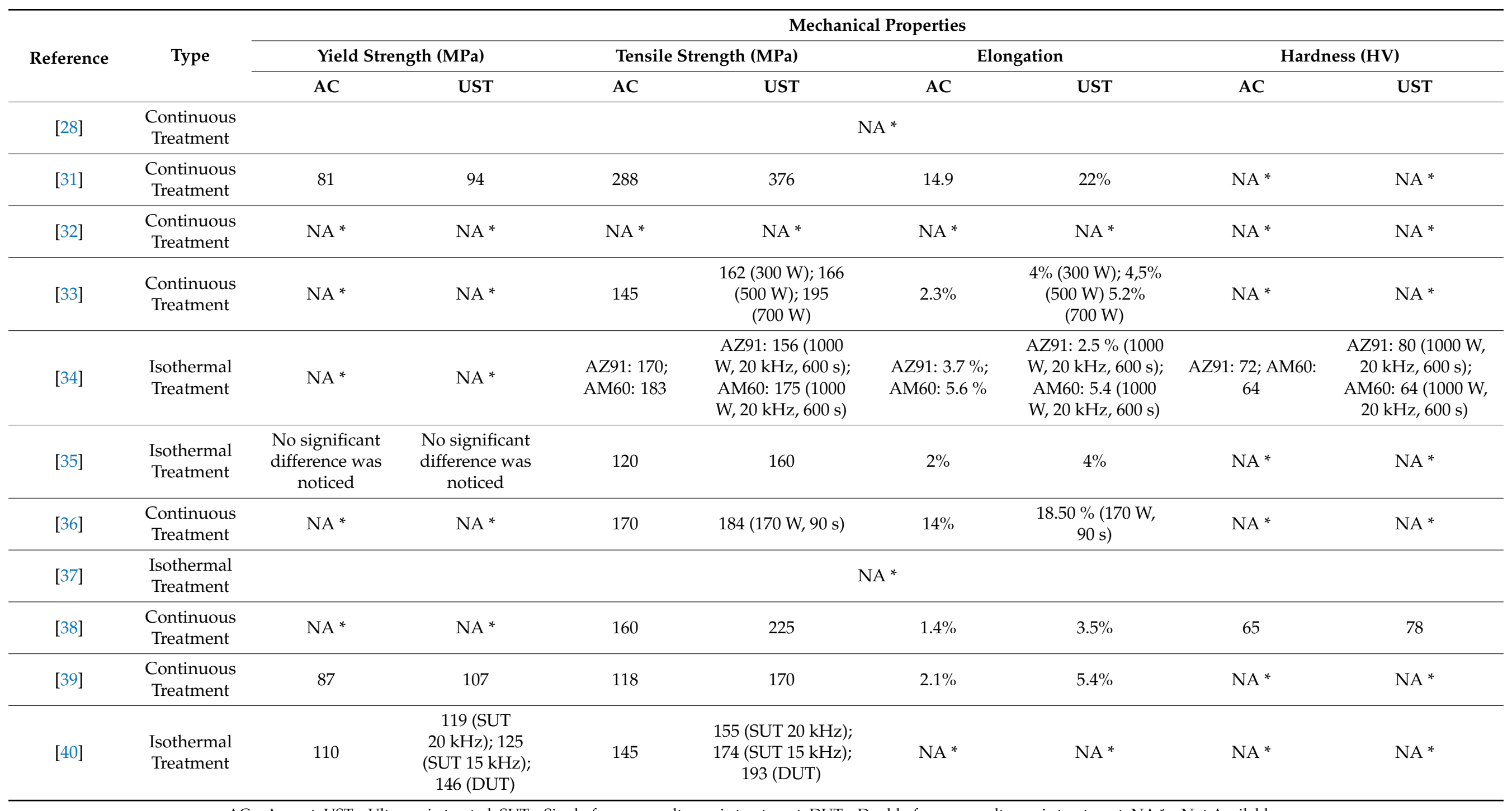


Table 6. Ultrasonic treatment mechanisms proposed by the authors of the included articles.

\begin{tabular}{|c|c|}
\hline Ultrasonic Treatment Mechanisms & References \\
\hline Increase of impurities wettability/ Heterogeneous nucleation & {$[3,6,8-10,31,32,34,35,37,38]$} \\
\hline $\begin{array}{l}\text { Promotion of the recent formed nuclei survival through increased } \\
\text { cooling rate due to (i) metal chill effect of the ultrasound horn and } \\
\text { (ii) streaming effect, which promotes the melt stirring and, thus, } \\
\text { its faster cooling }\end{array}$ & {$[10,31,33]$} \\
\hline $\begin{array}{l}\text { Increase of phases melting point promoted by the pressure pulse } \\
\text { caused by cavitation }\end{array}$ & {$[8,9,27,32]$} \\
\hline $\begin{array}{l}\text { Cavitation through undercooling verified at the melt/bubble } \\
\text { interface leading to nucleation of solid phase locally }\end{array}$ & {$[8,9,33,34]$} \\
\hline $\begin{array}{l}\text { Dispersion of the recent formed nuclei in the melt, enhancing } \\
\text { heterogeneous nucleation }\end{array}$ & {$[5,6,8,27,33,34,39]$} \\
\hline $\begin{array}{c}\text { Disintegration and distribution of the agglomerated nucleant } \\
\text { particles }\end{array}$ & {$[8,37]$} \\
\hline $\begin{array}{l}\text { Local melting of thinner parts and sharp edges leading to their } \\
\text { spheroidization }\end{array}$ & [8] \\
\hline Fragmentation of intermetallic phase & {$[38,39]$} \\
\hline Hard and soft impingement & [8] \\
\hline $\begin{array}{l}\text { Decrease of solute enrichment at the solidification front due to } \\
\text { high-speed flow originated by the collapse of cavitation bubbles }\end{array}$ & [39] \\
\hline Increase of cavitation area and initial cavitation nucleus & [37] \\
\hline $\begin{array}{l}\text { Fragmentation of the dendrites under the action of pressure } \\
\text { waves promoted by cavitation bubbles collapse }\end{array}$ & {$[38,39]$} \\
\hline $\begin{array}{l}\text { Mn-Al particles nucleation from the oxide followed by them } \\
\text { acting as nucleant for } \alpha \text {-Mg }\end{array}$ & [3] \\
\hline
\end{tabular}

\subsection{Treatment Parameters}

The conditions under which each study is carried out are the focus of most of the published work involving ultrasound for grain refinement. Parameters such as ultrasound power, processing time, and temperature at which treatment is performed are key factors that researchers have comprehensively investigated. The treatment conditions adopted by the authors of the articles considered in this review are presented in Tables 2 and 3.

Regarding the material used in the considered studies, AZ91 alloy was the most frequent choice $(11 / 17)$. Such a preference is justified by the popularity of this material for structural applications, despite its high susceptibility to solidification defects, low strength, and low ductility at room temperature $[27,35]$. Once these drawbacks are overcome through grain refinement, this alloy becomes an interesting option for studying ultrasound treatment effects [5]. AZ80 (4/17) and AZ31 (3/17) followed AZ91 in researchers' preference. Other authors' options included Mg-Li-Al, AJ62, and AM60 magnesium alloys with a single study each.

In the casting process, magnesium's high reactivity is a concern when melting it, given its tendency to oxidize and burn if no protection against oxidation is used. This behavior is due to the formation of a loose permeable oxide coating that allows oxygen passage, supporting burning below the oxide at the surface [41]. To prevent it, most authors resorted to a protective atmosphere composed of $\mathrm{CO}_{2}$ and $\mathrm{SF}_{6}(10 / 17)$, as $\mathrm{SF}_{6}$ presence promotes the formation of $\mathrm{MgFe}_{2}$ compound that blocks the pores of $\mathrm{MgO}$ film and renders it more protective against oxidation reactions [42]. Argon atmosphere was used in 2/17 studies while MAGREX flux and $\mathrm{LiCl}$ and $\mathrm{LiF}$ mixture were used in one work.

Of the included articles, 8/17 represent work related to the effect of the ultrasound treatment considering a single combination of treatment parameters. In contrast, the remaining ones sought the influence of a particular factor on the outcomes of the process. 
In this sense, several articles investigated the impact of ultrasound power $[8,27,33,36]$, duration $[3,9,34]$, and temperature $[9,10]$ of the treatment.

The temperature for ultrasonic vibration application varied from $605^{\circ} \mathrm{C}[9]$ to $740{ }^{\circ} \mathrm{C}$ [3] in the works that performed isothermal ultrasonication of the magnesium melt. It is noteworthy that a significant amount of these studies (4/8) used temperatures lesser than $650{ }^{\circ} \mathrm{C}$, which are close to the liquidus temperature of the alloys investigated. Such a condition is favorable to the cavitation-enhanced nucleation, but hinders the action of cavitation-induced dendrites fragmentation, which may be detrimental for grain refinement results [43].

Concerning the application of ultrasonic vibration to solidifying material, most studies reported performing the treatment during a pre-defined temperature range, despite that some authors did not detail the boundary values. Even so, according to the available information, 3/9 works applied the ultrasonic vibration down to a temperature lower than or close to liquidus one, from $571^{\circ} \mathrm{C}$ [39] for the AZ80 alloy to $608{ }^{\circ} \mathrm{C}$ [38] for the AZ91D alloy. One study stopped ultrasonication at a higher temperature of $680^{\circ} \mathrm{C}$ [28] using AZ31.

The ultrasonic power is a parameter that can also profoundly influence the results of material ultrasonication, regardless of the type of treatment applied. In this sense, different power values were reported, from $50 \mathrm{~W}$ [36] to $1000 \mathrm{~W}$ [27], which led to a different material response to the treatment. No relation appears to exist between the type of ultrasonic treatment and the range of ultrasonic power values applied.

The influence of the acoustic frequency applied was also a matter of study, although most authors chose one value between 19 and $20 \mathrm{kHz}(15 / 17)$. Chen et al. [34] followed a different approach and investigated the influence of the frequency used in the outcomes of melt treatment of AM60 and AZ91 alloys by comparing the results obtained using $15 \mathrm{kHz}$ and $20 \mathrm{kHz}$. Such an increase is suggested to be associated with a shorter period for the growth of the cavitation bubbles in the melt, which become smaller and thus may exhibit a more robust ability for degassing fine cavities on tiny particle surfaces, producing finer microstructure. An original proposal was explored by Ning et al. [40], who studied the effect of applying dual-frequency ultrasonic treatment to an AZ80 magnesium alloy melt and explained the experimental results in the light of the numerical ones obtained through simulation of the cavitation phenomenon.

Since molten magnesium does not have an affinity to iron and does not attack it, crucibles made of ferrous materials are often chosen to melt and hold magnesium alloys [41]. Hence, metallic crucibles, namely built-in steel, were used in $8 / 17$ of the works included herein, while alumina and graphite were chosen for $3 / 17$ and $2 / 17$ studies, respectively. No information was provided about the remaining studies. The ultrasonicated melt was poured into molds of different materials such as steel (2/8), copper (2/8), and sand $(1 / 8)$, which resulted in different cooling rates, or left within the crucible until complete solidification (2/8). Solidification within the crucible was the preferred approach of the researchers who studied the ultrasonication of solidifying material.

\subsection{Microstructural and Mechanical Characterization}

Microstructural characterization is perhaps the preferred approach to assess the effect of ultrasonic treatment. The description of the material's microstructure and the grain size and sphericity parameters provided by the authors is presented in Table 4 for each article considered in the review.

All the authors described the phases that were part of the samples, and a comparison between the untreated and treated pieces was presented in their articles. In this regard, the effect of ultrasound treatment on the morphology and size of $\alpha-\mathrm{Mg}$ was the focus of most of the studies included in this review (15/17), and only a few reported results concerning the modification of $\mathrm{Mg}_{17} \mathrm{Al}_{12}(7 / 17)$ and Al8Mn5 (2/17) intermetallic phases. Such a lack of research on this subject was already pointed out by Khosro Aghayani and Niroumand [8] and Yang et al. [27]. 
In the untreated condition, the $\alpha-\mathrm{Mg}$ phase presented coarse and non-uniform dendritic morphology, which was reported to be changed to a finer and more globular one after ultrasonic treatment by most of the authors. Still, Nie et al. [35] and Yang et al. [27] did not observe significant changes in the matrix configuration. This situation is suggested to be promoted by the large gap between treating temperature and melting point of the material, which complicates the survival of the newly formed particles that may remelt during the interval from the cessation of ultrasonication and pouring of the melt [27]. Moreover, the final grain size depends not only on the nucleation phenomenon but also on the growth condition. In this way, a slow latent heat extraction owing to a high mold temperature may hinder the refinement effect of the ultrasonic treatment, as reported by Nie et al. [35].

Another interesting finding was made by Qian et al. [28], who reported that the microstructural refinement was observed almost exclusively below the ultrasound radiating face. The grain refinement effect became less significant in other directions, and the grain size increased progressively with increasing distance to the radiating face. No noticeable grain refinement occurred adjacent to the cylindrical face of the sonotrode near the melt surface, despite its large chill surface and the melt surface disturbance triggered by transverse motion. In this sense, the authors proved that the immersion depth of the sonotrode into the melt had no considerable impact on its grain refinement effect. In a different study, Zhang et al. [6] found that, although the effect of cavitation diminishes away from the radiator, the whole ingots exhibited refined microstructure, suggesting that sufficient nuclei were transported and distributed in the bulk melt through acoustic streaming.

The fragmentation of the $\mathrm{Mg}_{17} \mathrm{Al}_{12}$ continuous network and its more uniform distribution along $\alpha-\mathrm{Mg}$ grain boundaries were the most significant changes concerning the effect of ultrasonic treatment on the morphology of this phase $[3,27,38,39]$. Additionally, a transition from coarse plates to fine and oriented lamellar morphology was reported by Nie et al. [35]. Remarkable is also the comprehensive study carried out by Khosro Aghayani and Niroumand [8], who identified three different intermetallic phases- $\mathrm{Mg}_{17} \mathrm{Al}_{12}, \mathrm{Mg}_{2} \mathrm{Si}$ and $\mathrm{MgFeAl}(\mathrm{Si})$ - and detailed the effect of the ultrasonic treatment on each one.

The grain size measurement was used in 12/17 articles as a criterion for assessing the treatment efficacy, while only $2 / 17$ chose to include the sphericity. Microstructural characterization was made, resorting primarily to optical (16/17) and scanning electron $(11 / 17)$ microscopy techniques.

The effect of ultrasonic power on the microstructure of the material was explored in the works of Gao et al. [33], Khosro Aghayani and Niroumand [8], Yao et al. [36], and Yang et al. [27]. Most results showed that the increase in ultrasonic power led to a decrease in grain size, which is explained by the associated intensification of the cavitation phenomenon. However, according to Shao et al. [37], there is a threshold value above which thermal effect compromised the grain refinement ability. The resultant decrease of the cooling rate of the melt may result in accelerating the grain growth and thus weakening the effect of grain refinement by ultrasonic treatment.

Regarding the duration of the treatment, longer treatments provide smaller grains, as stated in the works of Chen et al. [43], Patel et al. [9], and Emadi et al. [3].

The relation between the microstructure and mechanical properties of the material can be described by the Hall-Petch relation [44] as follows:

$$
\sigma_{y}=\sigma_{0}+k_{y} d^{-1 / 2}
$$

where $\sigma_{y}$ is the plastic flow stress [MPa], $\sigma_{0}$ is the friction stress of mobile dislocations [MPa], $k_{y}$ defines the characteristic constant that depends on the number of impurities and alloying elements [MPa. $\left.\mathrm{nm}^{1 / 2}\right]$, and $d$ is the grain size [nm]. In this sense, it is expected that the grain refinement effect of ultrasound may be reflected in enhancing the mechanical performance of the treated material, namely in its tensile properties. The mechanical properties described in each of the considered articles is presented in Table 5. 
From the considered studies, 9/17 included the mechanical characterization of both ultrasonically untreated and treated materials, of which 8 reported the tensile test results, and 1 presented those of the compression one. Only 2 works reported hardness results. All the authors reported the results of the samples without heat treatment.

The impact of the morphology of the phases on the materials' mechanical behavior was also discussed in some works (8/17).

In the untreated samples, a network of the brittle $\mathrm{Mg}_{17} \mathrm{Al}_{12}$ phase led to poor mechanical properties, namely low ultimate tensile strength and elongation. The refinement of this phase was suggested to be associated with a significant improvement of these properties [8]. Such a hypothesis was confirmed by the research of Nie et al. [35], who applied a solution heat treatment to a sample for dissolving $\mathrm{Mg}_{17} \mathrm{Al}_{12}$ and compared its mechanical performance with that of ultrasonically treated one. The similar results obtained by both samples confirmed that the reduction of $\mathrm{Mg}_{17} \mathrm{Al}_{12}$ continuity was behind the enhancement of the material mechanical behavior. Besides, the refinement of grains suppresses deformation by twinning and promotes deformation by sliding, leading to enhanced ductility [31,33]. The same authors also suggested that the absence of significant change in the yield properties of the ultrasonicated material was due to the weak effect that it had on refining the $\alpha-\mathrm{Mg}$ phase. This assumption is in agreement with the results presented by Hansong et al. [39], Ning et al. [40], and Emadi et al. [3], who achieved both finer microstructures and improved yield strength.

\subsection{Mechanisms behind Ultrasonic Refinement Ability}

Ultrasound consensual popularity for material processing stems from its remarkable ability to modify the different phases and tailor the microstructure in light alloys. Indeed, the control of the material solidification is crucial to enhance the mechanical performance, cast quality, and downstream processability of such alloys [45]. Despite that, the mechanisms that explain the ultrasonic refining effect are still the subject of several works, and a comprehensive explanation has not been formulated yet.

The material microstructure is complex and multiphase as it consists of primary grains and intermetallic and eutectic compounds. In this sense, the design of refining methods is of great scientific and technological importance, but it requires a deep knowledge of the material behavior, namely during its solidification [6].

Cavitation and acoustic streaming are frequently identified as the main mechanisms behind the efficacy of ultrasonic treatment regarding microstructure refinement. The cavitation phenomenon promotes a set of effects that may be associated with a more refined microstructure, namely: (1) formation of localized high-pressure points in the melt, which, according to the Clausius-Clapeyron equation, leads to the increase of its melting point, rendering significant localized undercooling and, therefore, intensified nucleation; (2) development of pressure pulses during the compression period of the acoustic wave as a result of the collapse of cavitation bubbles, promoting the rupture of large grains and dendritic arms as well as the disintegration of inoculation substrates clusters; (3) vaporization of the melt at the surface of the bubbles during the expansion period of the acoustic wave, leading to the decrease of the temperature at the interface where solid particles start nucleating; as soon as the bubbles collapse, the freshly formed particles are dispersed into the melt, and (4) increase of impurities and solid particles wettability, favoring heterogeneous nucleation. On the other hand, acoustic streaming, a liquid flow promoted by the acoustic pressure gradient, plays an essential function in developing mechanical forces that fragment the dendrite arms and disintegrate the particles agglomerates. Additionally, it is very effective for melt stirring [46].

The discussion of the mechanisms that underlie the ultrasound ability for refining the material's microstructure was reported by most of the works considered in this review (16/17). A summary of the proposed mechanisms is presented in Table 6. Accordingly, $10 / 17$ studies pointed to the acoustic streaming effect on modifying the material microstructure, and 9/17 studies proposed enhancing particles' wettability as the mechanism for 
the grain refining effect of ultrasonic treatment; $6 / 17$ articles mentioned the undercooling phenomenon resultant from cavitation.

Notwithstanding that the widespread role of cavitation and acoustic streaming on modifying the microstructure is well-accepted, the investigation of other contributions from nucleation, particle fragmentation, and coarsening is still scarce [6].

The temperature during cooling at which the different phases are formed defines the mechanisms through which ultrasonic treatment may modify the materials' microstructure [8]. In this sense, the processing temperature is a key parameter that may be chosen to enable the actuation of a particular mechanism or its effect on a specific phase.

Indeed, Patel et al. [9] applied ultrasonic vibration isothermally at $605^{\circ} \mathrm{C}$ and $620^{\circ} \mathrm{C}$, corresponding to liquidus temperature, to AS41 melt and differences were noticed regarding the material' microstructure. Ultrasonication above the liquidus temperature promoted the formation of equiaxed grains, while the application of the treatment below such temperature provided globular grains. Towards these results, the authors proposed different mechanisms for the refining effect of ultrasonic treatment according to the processing temperature. The application of ultrasonic vibration above the liquidus temperature is suggested to promote cavitation and therefore (i) enhance heterogeneous nucleation through the increase of melting point of the phases, (ii) improve the wettability of impurities and inclusions that become active in the solidification stage, and (iii) induce endothermic vaporization of liquid at the bubbles formed. At lower processing temperature, the authors proposed dendrites' fragmentation due to the cavitation bubbles' implosion as the mechanism behind the refinement effect, which is following the development of globular grains that have origin in fragmented dendrite arms.

In addition to the distinct mechanisms that act at different temperatures, the survival of the newly formed nuclei is deeply dependent on this parameter. In this regard, Nie et al. [35] found that although ultrasonic treatment had improved impurities' wettability and, therefore, favored heterogeneous nucleation, no significant refinement of the $\alpha-\mathrm{Mg}$ phase was observed. According to the authors, the remelting of the newly formed nuclei may be the reason for such results since the melt was elevated to a pouring temperature of $720{ }^{\circ} \mathrm{C}$ after ultrasonication.

A different result was presented by Khosro Aghayani and Niroumand [8] that identified the effect of ultrasonic cavitation on cleaning the poorly wetted surfaces as the primary mechanism behind the achievement of a significantly refined microstructure. Furthermore, they suggested that the cavitation and streaming phenomena also played an essential role in the disintegration and distribution of agglomerated nucleant particles. The increase of active nuclei led to earlier hard and soft impingement of the grains and the microstructure refinement.

Concerning intermetallic phases, the effect of ultrasonication is debated regarding mainly $\mathrm{Mg}_{17} \mathrm{Al}_{12}$ morphology. The melting point of the $\mathrm{Mg}_{17} \mathrm{Al}_{12}$ phase is $460{ }^{\circ} \mathrm{C}$ [47], which is far below the liquidus temperature of magnesium alloys. In this way, it is not expected that the pressure pulses may trigger such a high undercooling, which is the reason why acoustic streaming is suggested to be the dominant mechanism behind this phase refinement. In this regard, Yang et al. [27] indicated that the acoustic stream promotes the decrease of boundary segregation of aluminium and, hence, the reduction of aluminium concentration at the grain boundaries. Such an event leads then to the decrease of $\mathrm{Mg}_{17} \mathrm{Al}_{12}$ formation, according to the lever rule. Another interpretation was proposed by Khosro Aghayani and Niroumand [8], who identified the increased grain boundaries of $\alpha-\mathrm{Mg}$ and the more uniform chemical composition of the melt as the mechanisms behind the precipitation of $\mathrm{Mg}_{17} \mathrm{Al}_{12}$ phase at more locations, decreasing its continuity and size. Puga et al. [38] suggested fragmentation of $\mathrm{Mg}_{17} \mathrm{Al}_{12}$ phase under acoustic streaming as a promoter of intermetallic refinement, which was likely to happen once the authors applied the ultrasonic vibration to the mold during the material solidification.

The dynamic of $\mathrm{Al}_{8} \mathrm{Mn}_{5}$ phase formation under the action of ultrasonic vibration was described by Yang et al. [27], which applied ultrasonic vibration isothermally at $680^{\circ} \mathrm{C}$, 
about $20^{\circ} \mathrm{C}$ above the melting temperature of that phase. The authors suggested that the instantaneous pressure pulses promoted by cavitation could increase the melting point of the $\mathrm{Al}_{8} \mathrm{Mn}_{5}$ phase and, therefore, develop considerable local undercooling. In this way, the $\mathrm{Al}_{8} \mathrm{Mn}_{5}$ nuclei were formed and uniformly distributed through acoustic streaming, leading to a refined intermetallic phase. A similar explanation was proposed by Khosro Aghayani and Niroumand [8] for the refinement of $\mathrm{Mg}_{2} \mathrm{Si}$ and $\mathrm{MnFeAl}(\mathrm{Si})$ intermetallic phases when subjected to ultrasonic treatment. Additionally, the same authors also mentioned the local melt of thinner parts and sharper edges of these phases due to temperature increase during half-period compression of cavitation bubbles. Such a phenomenon not only increased the number of growing intermetallics but also promoted their spheroidization.

\subsection{Key Findings}

Table 7 presents, in chronological order, a summary of the objectives of the articles analyzed in this review and their key findings to provide the reader with an overview of studies' evolution in this field.

Table 7. Summary of the key findings reported in the included articles.

\begin{tabular}{|c|c|}
\hline Reference & Key Findings \\
\hline [27] & $\begin{array}{l}\text { - The microstructure of AZ91 alloy consisted of a matrix } \alpha-\mathrm{Mg} \text { phase, a dot-like } \mathrm{Al}_{8} \mathrm{Mn}_{5} \text { phase in matrix, a black } \\
\text { nodular } \mathrm{Mg}_{17} \mathrm{Al}_{12} \text { phase, and a netted-shape eutectic structure at grain boundaries. } \\
\text { - The grain size of } \alpha-\mathrm{Mg} \text { grains did not decrease significantly, and the average area of } \mathrm{Al}_{8} \mathrm{Mn}_{5} \text { particles } \\
\text { decreased to the minimum when the applied ultrasonic power was increased to } 600 \mathrm{~W} \text {. Further power increase } \\
\text { caused an inversion in such a tendency. } \\
\text { - The microstructure modifications were mainly associated with acoustic cavitation. } \\
\text { - The area percentage of } \mathrm{Mg}_{17} \mathrm{Al}_{12} \text { decreased gradually with increasing the applied ultrasonic power. } \\
\text { - The fraction change is mainly attributed to the acoustic streaming by reducing the boundary segregation of } \mathrm{Al} \text {. }\end{array}$ \\
\hline [3] & $\begin{array}{l}\text { - The ultrasonic treatment successfully refined the grains of the alloy. } \\
\text { - The application of ultrasound vibration for } 180 \mathrm{~s} \text { led to a significant decrease in the grain size (near } 75 \% \text { ) } \\
\text { compared with the untreated condition. This outcome was attributed to the cavitation related cleaning and } \\
\text { distribution of fine oxides as substrates for heterogeneous nucleation. } \\
\text { - The area fraction and average size of the deleterious } \mathrm{Mg}_{17} \mathrm{Al}_{12} \text { eutectics were decreased. This was thought to } \\
\text { be a side effect of the refined grains that resulted from ultrasonic treatment. } \\
\text { - Sonication of the melt resulted in increased area fraction, and improved distribution and spheroidization of the } \\
\text { Mn-Al intermetallics. This outcome was believed to directly affect ultrasonic treatment through improved } \\
\text { nucleation and cavitation-induced undercooling of the melt. } \\
\text { The sonication process enhanced the mechanical properties of the alloy. The tensile and yield strengths of the } \\
\text { alloy increased by } 17 \% \text {, and the ductility by } 63 \% \text {. Finer grain size, decreased } \mathrm{Mg}_{17} \mathrm{Al}_{12} \text { volume fraction, and } \\
\text { improved } \mathrm{Mn}-\mathrm{Al} \text { intermetallics distribution were thought to have contributed to this outcome. }\end{array}$ \\
\hline [6] & $\begin{array}{l}\text { - Ultrasonication during the solidification of Mg-alloys AZ31, AZ91, and AJ62 showed extensive grain } \\
\text { refinement. } \\
\text { The refinement effect was more intense near the radiator, where the grain size was smaller. The grain size } \\
\text { increased gradually as the distance increased. } \\
\text { A direct correlation between the average grain size and the solute growth restriction factor was observed in the } \\
\text { alloys indicating that strong fluid flow under ultrasonication does not diminish the growth restriction effect of } \\
\text { solute. } \\
\text { Grain refinement under ultrasonication was attributed to enhanced nucleation, indicating an increase in the } \\
\text { number and potency of nucleating agents. At the same time, eutectic and intermetallic modification was } \\
\text { suggested to be a growth-related phenomenon caused by coarsening and spheroidization under the strong } \\
\text { fluid flow from cavitation. }\end{array}$ \\
\hline
\end{tabular}


Table 7. Cont.

\begin{tabular}{|c|c|}
\hline Reference & Key Findings \\
\hline [8] & $\begin{array}{l}\text { - In this study, the effects of ultrasonic treatment on microstructural features and tensile strength of AZ91 } \\
\text { magnesium alloy were investigated. } \\
\text { The application of ultrasonic vibration to the melt before casting had a significant effect on the size and } \\
\text { sphericity of } \alpha \text {-Mg dendrites and the size, continuity, sphericity and distribution of intermetallic particles } \\
\text { formed during cooling and solidification of the alloy. } \\
\text { The increase of the ultrasonic power provided smaller, more rounded, and better-distributed grains and } \\
\text { intermetallic particles. } \\
\text { The microstructural effects were primarily associated with the cavitation and streaming phenomena during } \\
\text { ultrasonic treatment in the melt. } \\
\text { The ultrasonic treatment significantly enhanced the tensile strength of AZ91 alloy. Such effect was suggested to } \\
\text { be a consequence of discontinuity and refinement of } \mathrm{Mg}_{17} \mathrm{Al}_{12} \text { particles in these samples. }\end{array}$ \\
\hline [9] & $\begin{array}{l}\text { - In the ultrasonicated AS41 alloy, a nearly non-dendritic and refined microstructure was obtained instead of a } \\
\text { non-uniform dendritic structure of the untreated samples. } \\
\text { - When the ultrasonication was applied above the liquidus temperature, equiaxed grains formed, while globular } \\
\text { grains were obtained when the material was ultrasonicated below the liquidus temperature. } \\
\text { - The average grain size decreased with an increase in the treatment time. } \\
\text { - The } \mathrm{Mg}_{17} \mathrm{Al}_{12} \text { and } \mathrm{Mg}_{2} \mathrm{Si} \text { intermetallic phases were well-distributed along the grain boundaries after the } \\
\text { ultrasonic treatment. }\end{array}$ \\
\hline
\end{tabular}

- The decrease of the temperature at which the ultrasonic treatment led to the decrease of the nucleation temperature and promoted the increase of the undercooling for nucleation.

- The application of ultrasonication at temperatures from $615^{\circ} \mathrm{C}$ to $580^{\circ} \mathrm{C}$ and from $615^{\circ} \mathrm{C}$ to $595^{\circ} \mathrm{C}$ developed an undercooling smaller than that of the other tested conditions, and the difference between them was approximately $2{ }^{\circ} \mathrm{C}$.

[10] - The coarse dendrites formed with ultrasonic vibrations at temperatures below the liquidus temperature, while the finer microstructures were provided by ultrasonication during the nucleation stage.

- The mechanism of grain refinement was discussed based on the analyses of the solidification behavior under ultrasonic vibration. The authors suggested that a combination of enhanced heterogeneous nucleation and dendrite multiplication led to the grain refinement results obtained.

- Ultrasonic refinement occurred almost exclusively below the radiating face, and no refining effect was observed adjacent to the cylindrical face of the sonotrode immersed in the melt, despite providing a large chill surface and disturbing the melt surface through transverse motion.

- The immersion depth of the sonotrode in the melt has no noticeable impact on the resulting ultrasonic

[28] - The finest grain size resulting from ultrasonication occurred immediately below the radiating face, and the grain size increased progressively with increasing distance from the radiating face towards the crucible walls.

- Ultrasonic refinement was symmetrical about the principal ultrasound propagation direction. The grain size along the principal propagation direction was progressively smaller than other directions at the same distance from the radiating face.

- On average, the grain density of the ultrasonicated material increased by a factor of $8 \times 10^{3}$ compared with the untreated one. According to the authors, such a significant increase in grain density is associated with a

[32] dramatic increase in active nucleants.

- In addition to the increase of the grain density, the presence of uniform equiaxed grains suggests that the grain refinement achieved is more likely due to enhanced nucleation than dendrite fragmentation.

- The increase of the ultrasonic power used in the treatment of the material during its solidification led to the increase of both nucleation and cooling rates and, consequently, promoted the refinement of AZ91

[33] microstructure.

- $\quad$ The coarse dendritic microstructure of the untreated material was gradually transformed into a more globular and finer one as the ultrasonic power increased. 
Table 7. Cont

\begin{tabular}{|c|c|}
\hline Reference & Key Findings \\
\hline [34] & $\begin{array}{l}\text { - Ultrasonic treatment of the melt is a new approach for magnesium and magnesium alloy casting. The } \\
\text { fundamental basis is the generation of cavitation bubbles during ultrasonic treatment of the melt, which } \\
\text { induces dispersion and degassing action. } \\
\text { The application of ultrasonic vibration promoted the refinement of the eutectic phase, the disruption of the } \\
\text { oxide films, and the reduction of the inclusion particles size. } \\
\text { - Ultrasonic treatment had a grain-refining effect on cast magnesium and AZ91 and AM60 magnesium alloys, } \\
\text { but no improvement was observed regarding their mechanical properties. } \\
\text { - The effects of grain refinement increased with increasing acoustic frequency, acoustic power, and treatment } \\
\text { time. } \\
\text { The results of grain refinement gradually faded with increased settling time of the melts after ultrasonic } \\
\text { treatment. }\end{array}$ \\
\hline [35] & $\begin{array}{l}\text { - The effect of ultrasonic vibration and solution heat treatment on microstructures and tensile properties of } \mathrm{AZ91} \\
\text { alloy was experimentally investigated. } \\
\text { Ultrasonic vibration had a substantial effect on the sphericity and distribution of the phase } \mathrm{Mg}_{17} \mathrm{Al}_{12} \text { formed } \\
\text { during the cooling and solidification of the alloy. } \\
\text { - The solution heat treatment dissolved the phase } \mathrm{Mg}_{17} \mathrm{Al}_{12} \text { in both the alloy with ultrasonic vibration and } \\
\text { without ultrasonic vibration. } \\
\text { AZ91 samples subjected to ultrasonic vibration exhibited improved tensile properties compared with } \\
\text { - Thtreated ones. } \\
\text { the effect of solution heat treatment on the morphology of the phase } \mathrm{Mg}_{17} \mathrm{Al}_{12} \text { in the alloy can improve the } \\
\text { tensile properties. Such results suggest that the } \mathrm{Mg}_{17} \mathrm{Al}_{12} \text { phase precipitated at grain boundaries may be } \\
\text { detrimental to the mechanical performance of } \mathrm{AZ91} \text { alloy. }\end{array}$ \\
\hline [36] & $\begin{array}{l}\text { - } \mathrm{Mg}-8 \mathrm{Li}-3 \mathrm{Al} \text { alloy microstructure was composed of } \alpha \text { and } \beta \text { phases. } \alpha \text {-phase was modified from a coarse } \\
\text { rosette-like structure to a finely globular one with ultrasonic vibration. The finely rounded structure was } \\
\text { obtained, especially when the power was } 170 \mathrm{~W} \text {. This effect became better as the duration of the treatment } \\
\text { increased. } \\
\text { Improvements of } 9.5 \% \text { and } 45.7 \% \text { were achieved in the tensile strength and elongation, respectively, when } \\
\text { ultrasonic treatment was applied at } 170 \mathrm{~W} \text { during } 90 \mathrm{~s} \text {. }\end{array}$ \\
\hline [37] & $\begin{array}{l}\text { - Ultrasonic treatment of the melt refined the microstructure of the AZ80 alloy. } \\
\text { - The authors suggested that such an effect was due to both cavitation and streaming phenomena. } \\
\text { - The increase of ultrasonic power from } 0 \mathrm{~W} \text { to } 600 \mathrm{~W} \text { led to a significant decrease in the grain size, but such a } \\
\text { tendency changed when the power increased further. } \\
\text { - The optimal ultrasonic power for the ultrasonic grain refinement of the AZ80 alloy was } 600 \mathrm{~W} \text {. }\end{array}$ \\
\hline [38] & $\begin{array}{l}\text { - The application of high-intensity acoustic vibration promoted a uniform dispersion of the } \beta-\mathrm{Mg}_{17} \mathrm{Al}_{12} \\
\text { intermetallic phase and reduced the level of porosity. } \\
\text { Both tensile strength and strain were increased by } 40.7 \% \text { and } 150 \% \text {, respectively, when the material was } \\
\text { processed through ultrasound vibration applied to the mold. } \\
\text { - Ultrasonic treatment in the AZ91D samples suggested improving static mechanical properties without } \\
\text { compromising the dynamic mechanical properties of these alloys. }\end{array}$ \\
\hline [39] & $\begin{array}{l}\text { - The microstructure of AZ80 alloy was remarkably improved after ultrasonic melt treatment. The primary } \alpha-\mathrm{Mg} \\
\text { phase was changed from coarse dendrites to refined equiaxed grains, and the continuous brittle } \beta-\mathrm{Mg}_{17} \mathrm{Al}_{12} \\
\text { phase at } \alpha-\mathrm{Mg} \text { boundaries was refined and became discontinuous. } \\
\text { Ultrasonic treatment decreased the segregation of aluminium element at the grain boundaries, and more } \\
\text { aluminium element dissolved into the } \alpha-\mathrm{Mg} \text { matrix. } \\
\text { The tensile properties of } \mathrm{AZ} 80 \text { alloy with ultrasonic treatment were significantly improved compared with } \\
\text { those of the untreated alloy. Such an achievement was mainly related to the nearly equiaxed microstructure } \\
\text { and dispersed } \beta-\mathrm{Mg}_{17} \mathrm{Al}_{12} \text { phase the grain boundaries. }\end{array}$ \\
\hline
\end{tabular}


Table 7. Cont.

\begin{tabular}{l}
\hline Reference \\
\hline - The effect of refinement of ultrasonic is better than conventional casting. The $15 \mathrm{kHz}$ SUT refinement is greater \\
than $20 \mathrm{kHz}$, and the refinement of $15+20 \mathrm{kHz}$ DUT is better. \\
The samples treated under DUT exhibited the highest tensile strength, followed by $15 \mathrm{kHz}$ SUT and $20 \mathrm{kHz}$ \\
SUT. \\
- $\begin{array}{l}\text { Numerical studies performed by the authors showed that the cavitation area gradually increased with the } \\
\text { increase of the initial cavitation nucleus radius. In the range of initial cavitation radius, the cavitation area } \\
\text { produced by } 15 \mathrm{kHzSUT} \text { was approximately twice that of SUT } 20 \mathrm{kHz} \text {; after applying DUT, changing of the } \\
\text { spatial distribution of cavitation area and the increasing of cavitation area were caused by nonlinear } \\
\text { superposition, and the cavitation area was more significant than the sum of the two SUT cavitation areas, } \\
\text { which justifies the improved results. }\end{array}$ \\
\hline 40$] \quad$
\end{tabular}

\section{Conclusions}

This systematic review on the application of ultrasonic treatment in magnesium alloys is valuable to synthesize the work done in this research field and bridge the gap between new researchers and the previously developed approaches so new investigation opportunities may be identified.

The articles considered in this review were categorized in isothermal and continuous ultrasonic treatment since these were the main approaches pursued by the authors.

The main conclusions of this review are as follows:

- No significant preference was found for the application of isothermal or continuous ultrasonic treatment.

- AZ91 alloy is the most used material, which is due to its popularity for structural applications.

- Given the magnesium tendency to oxidize and burn, most authors resorted to controlled atmospheres. The more common option is the use of $\mathrm{SF}_{6}+\mathrm{CO}_{2}$ protective atmosphere.

- The temperature for ultrasonic vibration application varied from $605{ }^{\circ} \mathrm{C}$ to $740{ }^{\circ} \mathrm{C}$ in the works that performed isothermal ultrasonication of the magnesium melt. A significant part of these studies used temperatures lesser than $650{ }^{\circ} \mathrm{C}$, which are close to the liquidus temperature of the alloys investigated. Regarding the continuous application of ultrasonic treatment during the material's solidification, the majority of the studies reported performing the treatment during a pre-determined temperature range.

- Some authors investigated the effect of ultrasonic power on the resultant microstructure. It is suggested that the increase of ultrasonic power leads to the enhancement of ultrasonic ability to produce finer microstructures despite there is a threshold for such a tendency due to thermal effect.

- Most studies used an ultrasonic frequency between 19 and $20 \mathrm{kHz}$. Recent work on the application of dual-frequency ultrasonic treatment provided promising results, which is the reason why this is a potential area of research.

- $\quad$ The $\alpha$-Mg phase is reported to be refined and changed to more globular morphology after ultrasonic treatment. However, the temperature at which the melt is treated and poured is critical for recently formed nuclei survival.

- There is a lack of research concerning the effect of ultrasonic on the intermetallic phase's morphology. Some authors reported that $\mathrm{Mg}_{17} \mathrm{Al}_{12}$ became smaller and more discontinuous after the application of ultrasonic treatment under certain conditions.

- Tensile properties of ultrasonicated material were found to be enhanced. Such achievement is associated mainly with the smaller grain size and the refinement of $\mathrm{Mg}_{17} \mathrm{Al}_{12}$, a brittle phase with a deleterious effect on the material's mechanical performance. Few works report the effect on the material's hardness, which is increased after ultrasonication.

- Mechanisms behind ultrasound ability for grain refinement is dependent on the temperature at which the treatment is performed. Acoustic cavitation and streaming 
are suggested as the main phenomena that lead to finer and more homogenous microstructure. Dendrite fragmentation and remelt of thinner parts and sharp edges are also suggested to play a role in the spheroidization of the phases. The study of the effect of ultrasonication on the material's solidification behavior, namely regarding the undercooling phenomenon, is a research topic that deserves researchers' attention once it may bring to light more information about the underlying mechanisms of microstructure refinement.

The grain refinement of magnesium alloys is a hot topic that brings together the interest of both academic and industrial researchers. Indeed, the results obtained mainly at laboratory scale may be interpreted with optimism regarding its scale-up to industrial environment. In this sense, some investigation may be carried out to comprehensively understand the results that it may provide and the yet unexplored potential of this processing technique. Future work must encompass the study of the effect of ultrasonication of large magnesium melts given that it may bring important challenges due to the different processing conditions. Furthermore, the impact of the ultrasound treatment on the corrosion behavior of the material is a topic that must also be addressed since most magnesium applications are sensitive to such a phenomenon. Furthermore, the study of the influence of ultrasonication on the material's microstructure may bring important information to clarify the latter's role on the mechanical behavior and shed light on different deformation mechanisms. Such knowledge is of great value to improve the downstream processability of magnesium alloys.

Author Contributions: I.V.G.: conceptualization, methodology, formal analysis, data curation, writing - original draft preparation, writing — review and editing H.P.: conceptualization, methodology, validation, writing — original draft preparation, supervision, project administration, funding acquisition J.L.A.: conceptualization, validation, writing-review and editing, supervision. All authors have read and agreed to the published version of the manuscript.

Funding: This work was supported by national funds supported this work through FCT-Portuguese Foundation for Science and Technology on the aim of the research doctoral Grant PD/BD/140094/2018 and by FEDER funds through the COMPETE program with the reference project PTDC/SEMTEC/ $3827 / 2014$. Additionally, this work was supported by FCT national funds, under the national support to R\&D units grant, through the reference project UIDB/04436/2020.

Institutional Review Board Statement: Not applicable.

Informed Consent Statement: Not applicable.

Data Availability Statement: Not applicable.

Conflicts of Interest: The authors declare no conflict of interest.

\section{References}

1. Kulekci, M.K. Magnesium and its alloys applications in automotive industry. Int. J. Adv. Manuf. Technol. 2007, 39, 851-865. [CrossRef]

2. Dziubińska, A.; Gontarz, A.; Dziubiński, M.; Barszcz, M. The forming of magnesium alloy forgings for aircraft and automotive applications. Adv. Sci. Technol. Res. J. 2016, 10, 158-168. [CrossRef]

3. Emadi, P.; Ravindran, C. The influence of high temperature ultrasonic processing time on the microstructure and mechanical properties AZ91E magnesium alloy. J. Mater. Eng. Perform. 2021, 30, 1188-1199. [CrossRef]

4. Kojima, Y. Platform science and technology for advanced magnesium alloys. Project of platform science and technology for advanced magnesium alloys. Mater. Trans. 2001, 42, 1154-1159. [CrossRef]

5. Friedrich, H.E.; Mordike, B.L. Magnesium Technology (Metallurgy, Design Data, Applications); Springer International Publishing: Berlin, Germany, 2006.

6. Zhang, X.; Kotadia, H.R.; Depner, J.; Qian, M.; Das, A. Effect of ultrasonication on the solidification microstructure in al and MG-alloys. In Light Metals 2019; Chesonis, C., Ed.; Springer International Publishing: Cham, Switzerland, 2019; pp. 1589-1595. [CrossRef]

7. Yang, Y.; Xiong, X.; Chen, J.; Peng, X.; Chen, D.; Pan, F. Research advances in magnesium and magnesium alloys worldwide in 2020. J. Magnes. Alloy. 2021, 9, 705-747. [CrossRef] 
8. Aghayani, M.K.; Niroumand, B. Effects of ultrasonic treatment on microstructure and tensile strength of AZ91 magnesium alloy. J. Alloy. Compd. 2011, 509, 114-122. [CrossRef]

9. Patel, B.; Chaudhari, G.; Bhingole, P. Microstructural evolution in ultrasonicated AS41 magnesium alloy. Mater. Lett. 2012, 66, 335-338. [CrossRef]

10. Liu, X.; Osawa, Y.; Takamori, S.; Mukai, T. Grain refinement of AZ91 alloy by introducing ultrasonic vibration during solidification. Mater. Lett. 2008, 62, 2872-2875. [CrossRef]

11. Kwak, T.; Kim, W. Hot compression behavior of the $1 \mathrm{wt} \%$ calcium containing Mg-8Al-0.5Zn (AZ80) alloy fabricated using electromagnetic casting technology. Mater. Sci. Eng. A 2014, 615, 222-230. [CrossRef]

12. Easton, M.A.; Davies, C.; Barnett, M.R.; Pravdic, F. Effect of solidification grain refinement on the development of wrought MG alloys. Mater. Sci. Forum 2007, 539-543, 1729-1734. [CrossRef]

13. Qian, M.; Ramirez, A.; Das, A.; StJohn, D. The effect of solute on ultrasonic grain refinement of magnesium alloys. J. Cryst. Growth 2010, 312, 2267-2272. [CrossRef]

14. Jiang, L.; Liu, W.; Wu, G.; Ding, W. Effect of chemical composition on the microstructure, tensile properties and fatigue behavior of sand-cast Mg-Gd-Y-Zr alloy. Mater. Sci. Eng. A 2014, 612, 293-301. [CrossRef]

15. Li, Z.; Chen, M.; Li, W.; Zheng, H.; You, C.; Liu, D.; Jin, F. The synergistic effect of trace Sr and Zr on the microstructure and properties of a biodegradable Mg-Zn-Zr-Sr alloy. J. Alloy. Compd. 2017, 702, 290-302. [CrossRef]

16. Nagasivamuni, B.; Wang, G.; StJohn, D.; Dargusch, M. Effect of ultrasonic treatment on the alloying and grain refinement efficiency of a Mg-Zr master alloy added to magnesium at hypo- and hyper-peritectic compositions. J. Cryst. Growth 2019, 512, 20-32. [CrossRef]

17. StJohn, D.H.; Qian, M.; Easton, M.; Cao, P.; Hildebrand, Z. Grain refinement of magnesium alloys. Met. Mater. Trans. A 2005, 36, 1669-1679. [CrossRef]

18. Li, M.; Tamura, T.; Omura, N.; Murakami, Y.; Tada, S. Grain refinement of AZCa912 alloys solidified by an optimized electromagnetic stirring technique. J. Mater. Process. Technol. 2016, 235, 114-120. [CrossRef]

19. Balasubramani, N.; Wang, G.; StJohn, D.H.; Dargusch, M.S. The poisoning effect of AL and be on MG-1 wt.\% ZR alloy and the role of ultrasonic treatment on grain refinement. Front. Mater. 2019, 6. [CrossRef]

20. Emley, E.F. Principles of Magnesium Technology; Pergamon Press: Oxford, UK, 1966.

21. Polmear, I.J. Recent developments in light alloys. Mater. Trans. JIM 1996, 37, 12-31. [CrossRef]

22. Pekguleryuz, M.O.; Baril, E. Development of Creep Resistant Mg-Al-Sr Alloys. Essent. Read. Magnes. Technol. 2016, 283-289. [CrossRef]

23. Guo, H.-M.; Zhang, A.-S.; Hu, B.; Ding, Y.; Liu, X.-B. Refining microstructure of AZ91 magnesium alloy by introducing limited angular oscillation during initial stage of solidification. Mater. Sci. Eng. A 2012, 532, 221-229. [CrossRef]

24. Li, M.; Tamura, T.; Miwa, K. Controlling microstructures of AZ31 magnesium alloys by an electromagnetic vibration technique during solidification: From experimental observation to theoretical understanding. Acta Mater. 2007, 55, 4635-4643. [CrossRef]

25. Gao, Y.-L.; Li, Q.-S.; Gong, Y.-Y.; Zhai, Q.-J. Comparative study on structural transformation of low-melting pure Al and high-melting stainless steel under external pulsed magnetic field. Mater. Lett. 2007, 61, 4011-4014. [CrossRef]

26. Zhao, J.; Cheng, Y.; Han, K.; Zhang, X.; Xu, Z.; Zhai, Q. Numerical and experimental studies of surface-pulsed magneto-oscillation on solidification. J. Mater. Process. Technol. 2015, 229, 286-293. [CrossRef]

27. Yang, Y.-S.; Wang, J.-C.; Wang, T.; Liu, C.-M.; Zhang, Z.-M. Effects of ultrasonic treatment on microstructures of AZ91 alloy. Trans. Nonferrous Met. Soc. China 2014, 24, 76-81. [CrossRef]

28. Qian, M.; Ramirez, A.; Das, A. Ultrasonic refinement of magnesium by cavitation: Clarifying the role of wall crystals. J. Cryst. Growth 2009, 311, 3708-3715. [CrossRef]

29. Garamus, V.; Limberg, W.; Serdechnova, M.; Mei, D.; Lamaka, S.; Ebel, T.; Willumeit-Römer, R. Degradation of titanium sintered with magnesium: Effect of hydrogen uptake. Metals 2021, 11, 527. [CrossRef]

30. Czerwinski, F. Modern aspects of liquid metal engineering. Met. Mater. Trans. A 2016, 48, 367-393. [CrossRef]

31. Liu, X.; Osawa, Y.; Takamori, S.; Mukai, T. Microstructure and mechanical properties of AZ91 alloy produced with ultrasonic vibration. Mater. Sci. Eng. A 2008, 487, 120-123. [CrossRef]

32. Ramirez, A.; Qian, M.; Davis, B.; Wilks, T.; StJohn, D. Potency of high-intensity ultrasonic treatment for grain refinement of magnesium alloys. Scr. Mater. 2008, 59, 19-22. [CrossRef]

33. Gao, D.; Li, Z.; Han, Q.; Zhai, Q. Effect of ultrasonic power on microstructure and mechanical properties of AZ91 alloy. Mater. Sci. Eng. A 2009, 502, 2-5. [CrossRef]

34. Chen, Y.-J.; Hsu, W.-N.; Shih, J.-R. The effect of ultrasonic treatment on microstructural and mechanical properties of cast magnesium alloys. Mater. Trans. 2009, 50, 401-408. [CrossRef]

35. Nie, K.; Wang, X.; Wu, K.; Zheng, M.; Hu, X. Effect of ultrasonic vibration and solution heat treatment on microstructures and tensile properties of AZ91 alloy. Mater. Sci. Eng. A 2011, 528, 7484-7487. [CrossRef]

36. Yao, L.; Hao, H.; Ji, S.-H.; Fang, C.-F.; Zhang, X.-G. Effects of ultrasonic vibration on solidification structure and properties of Mg-8Li-3Al alloy. Trans. Nonferrous Met. Soc. China 2011, 21, 1241-1246. [CrossRef]

37. Shao, Z.; Le, Q.; Zhang, Z.; Cui, J. Effect of ultrasonic power on grain refinement and purification processing of AZ80 alloy by ultrasonic treatment. Met. Mater. Int. 2012, 18, 209-215. [CrossRef] 
38. Puga, H.; Carneiro, V.; Barbosa, J.; Vieira, V. Effect of ultrasonic treatment in the static and dynamic mechanical behavior of AZ91D MG alloy. Metals 2015, 5, 2210-2221. [CrossRef]

39. Hansong, X.; Di, L.; Gang, Y.; Dingfei, Z. Ultrasonic effects on microstructure evolution and mechanical properties of AZ80 magnesium alloy. Rare Met. Mater. Eng. 2016, 45, 1401-1405. [CrossRef]

40. Ning, S.; Chen, X.; Le, Q.; Wang, H.; Hu, C. Effect of ultrasonic frequency on cavitation behavior, microstructure and mechanical properties of AZ80 magnesium alloy. Mater. Res. Express 2019, 6, 0865d9. [CrossRef]

41. Luo, A.A. Magnesium casting technology for structural applications. J. Magnes. Alloy. 2013, 1, 2-22. [CrossRef]

42. King, J.F. Environmental Challenges Facing the Magnesium Industry-SF6 Replacement. In Proceedings of the 60th Annual World Magnesium Conference, Wauconda, IL, USA, 11-12 May 2003.

43. Chen, X.; Le, Q. Application of ultrasonics on preparation of magnesium alloys. In Sonochemical Reactions; Karakuş, S., Ed.; Intechopen: London, UK, 2020. [CrossRef]

44. Ono, N.; Nowak, R.; Miura, S. Effect of deformation temperature on Hall-Petch relationship registered for polycrystalline magnesium. Mater. Lett. 2004, 58, 39-43. [CrossRef]

45. Spittle, J.A. Columnar to equiaxed grain transition in as solidified alloys. Int. Mater. Rev. 2006, 51, 247-269. [CrossRef]

46. Ma, L.; Chen, F.; Shu, G. Preparation of fine particulate reinforced metal matrix composites by high intensity ultrasonic treatment. J. Mater. Sci. Lett. 1995, 14, 649-650. [CrossRef]

47. Nayeb-Hashemi, A.A.; Clark, J.B. Phase Diagrams of Binary Magnesium Alloys; ASM International: Metals Park, OH, USA, 1988. 